\title{
A compilation of energy costs of physical activities
}

\author{
Mario Vaz ${ }^{1, *}$, Nadine Karaolis ${ }^{2}$, Alizon Draper $^{2}$ and Prakash Shetty ${ }^{2}$ \\ 'Division of Nutrition, Department of Physiology, St. John's Medical College, Bangalore 560034, India: \\ ${ }^{2}$ Public Health Nutrition Unit, London School of Hygiene and Tropical Medicine, 49/51 Bedford Square, London \\ WC1B 3DP, UK
}

\begin{abstract}
Objectives: There were two objectives: first, to review the existing data on energy costs of specified activities in the light of the recommendations made by the Joint Food and Agriculture Organization/World Health Organization/United Nations University (FAO/WHO/UNU) Expert Consultation of 1985. Second, to compile existing data on the energy costs of physical activities for an updated annexure of the current Expert Consultation on Energy and Protein Requirements.

Design: Electronic and manual search of the literature (predominantly English) to obtain published data on the energy costs of physical activities. The majority of the data prior to 1955 were obtained using an earlier compilation of Passmore and Durnin. Energy costs were expressed as physical activity ratio (PAR); the energy cost of the activity divided by either the measured or predicted basal metabolic rate (BMR). Results: The compilation provides PARs for an expanded range of activities that include general personal activities, transport, domestic chores, occupational activities, sports and other recreational activities for men and women, separately, where available. The present compilation is largely in agreement with the 1985 compilation, for activities that are common to both compilations.

Conclusions: The present compilation has been based on the need to provide data on adults for a wide spectrum of human activity. There are, however, lacunae in the available data for many activities, between genders, across age groups and in various physiological states.
\end{abstract}

\author{
Keywords \\ Physical activity \\ Energy expenditure
}

\section{Background}

The 1985 Joint Food and Agriculture Organization/World Health Organization/United Nations University (FAO/ WHO/UNU) Expert Consultation on Energy and Protein Requirements drew up a list of gross energy expenditure in specified activities (Annex 5, of the Technical Report Series, 724) ${ }^{1}$. Energy expenditure for each activity was expressed in terms of a 'metabolic constant', a multiple of basal metabolic rate (BMR). Separate lists were drawn up for male and female adults, for both developing and developed countries and in various categories of activity.

\section{Aim of the present compilation of energy costs in specified activities}

The aim of the present exercise was:

1. To review the existing data on energy costs of specified activities in the light of the recommendations made by the Joint $\mathrm{FAO} / \mathrm{WHO} / \mathrm{UNU}$ Expert Consultation of $1985^{1}$.

2. To compile existing data into an annexure for the present expert consultation on energy and protein requirements.

\section{Methodology used}

Both electronic and manual searches were employed in order to obtain published data on the energy costs of physical activities. The vast majority of data before 1955 have been obtained from the compilation of Passmore and Durnin $^{2}$. Most of the subsequent literature has dealt with energy costs of activity under constrained laboratory conditions using treadmill or bicycle ergometry protocols. Another large section of literature has focused on the energy costs of sports, primarily in well-trained and elite athletes. The 1985 Expert Consultation list of the energy costs of specified activities, focused primarily on daily activities of individuals and this has also been the focus of the present compilation.

Studies that were included in the final analysis were required to have:

- Measurements of the energy costs of activities. The vast majority of studies included had measurements using the Douglas bag technique or using the Kofranyi Michaelis instrument. Some studies reported energy expenditure in terms of oxygen consumption.

- Measurements of BMR, or alternatively, have sufficient anthropometric details to allow for the prediction of BMR. 
- Data presented in the accompanying tables which for the main part, are the primary sources of the compilation of energy costs, include a variety of information:

- The source of the data.

- The characteristics of the subjects.

- The nature of the activity, with details wherever available.

- The instrumentation used in the measurements.

- The duration of measurement, wherever available.

- The original data of the energy costs of the activities studied.

In addition, the following data have also been computed and added to the data of each study:

- Predicted BMR: for all studies that did not actually measure BMR, BMR was computed using the FAO/ WHO/UNU prediction equations of $1985^{1}$. The equations used were those that either had height and weight as predictor variables or weight alone, depending on the availability of the anthropometric data.

- Physical activity ratios (PARs): otherwise referred to as 'metabolic constant' in the 1985 compilation, was computed as the energy cost of the activity divided by either the predicted or measured BMR.

- Energy costs of activities: wherever data was presented in the form of oxygen consumption, this was converted into energy equivalents using a standard 1 litre $\mathrm{O}_{2}=5 \mathrm{kcal}$.

- The final collation of the energy costs of specified activities includes the data of the present compilation as well as some data of the 1985 compilation for which data was not reviewed in the present compilation. Where more than one study has contributed to the PAR of a specific activity, a 'PAR range' has been provided. This essentially indicates the highest and lowest reported PAR for a particular activity. This provides the user of the data with some idea of the betweenstudy variability in the estimation of the PAR.

\section{Comparison of the present compilation with the 1985 data}

The present compilation is largely in agreement with the 1985 data for activities that are common to both compilations. In some situations, despite the apparently common source of information, calculated PARs differ marginally. This is likely to be due to different methods of computing the BMR. In the final compilation of the data, PARs are reported based on the calculations done during this compilation, even when the differences between the previous and the present compilation appear small. The final decision on which of the PARs to use should take into account the different methods, if any, of arriving at the PAR and of usage of BMR prediction equations. The methods by which the original PARs in the 1985 compilation were arrived at were unavailable to the authors at the time of this compilation. One PAR that has been retained from the original compilation despite the fact that the actual PAR may be marginally lower is that of sleep, which has been retained as 1.0. This is because of the greater ease with which the total energy cost of sleep can be computed and the marginal effect that changing the PAR would have on the estimate of daily physical activity level (PAL). For some of the activities, the present compilation appears more than marginally different from the 1985 recommendations. This may be due to the addition of more studies in the present compilation and the high variability of PARs across studies.

\section{Limitations of the data}

This compilation uses data from many sources and the studies that have been reviewed have varied in their detail and methodology. Stringent selection criteria, would have reduced the available data on PARs considerably. A decision was taken to be inclusive, rather than exclusive, with the aim of providing a wide coverage of activities. Wherever possible, the data were assessed for face validity when more common activities, the PARs of which have been better documented, were described. Nevertheless, the user of the tables should be aware of the issues that need to be taken into consideration while accepting the data. These include:

- Varied descriptions of the activities: while some studies have been explicit in the description of the activities, most have used single word or short phrase descriptors. This poses a problem while collating data on the same activity from multiple sources. In the present compilation, the descriptors used by the investigators have largely been conserved and in situations where the same activity may have been performed differently (using a different posture, for instance), the activity is listed separately with its own PAR. This does not necessarily mean that the two activities have truly different PARs, merely that one descriptor may be more applicable in a local situation than another. For instance, under 'domestic chores', PARs for 'washing clothes' have been listed under several heads: 'washing clothessquatting on the ground', 'washing clothes-standing', 'washing clothes-sitting', 'washing clothes-unspecified' and 'washing small clothes'. The advantage here for the user is that they can choose which activity is most suitable for their local circumstances, rather than have to make a decision as to whether a generic 'washing clothes' is applicable for them.

- Varied methodology used: some studies have used measurements at 'steady-state', others have used 'point' measurements. The criteria for 'steady-state' measurements also vary between studies. Details about the 
calibration of instruments are variable across different studies.

- Computation of the BMR: in the present compilation, computation of BMRs was done using the anthropometry provided. While in all instances the BMR was computed using the $\mathrm{FAO} / \mathrm{WHO} / \mathrm{UNU}$ equations of 1985, anthropometric data was often available only for the entire study group, while the specified activities were measured in subsets of the whole group. This would have contributed to errors in the computation of the BMR for the subset and estimation of the PAR.

- Conditions of measurement are frequently inadequately described. Details like the climatic conditions, the time of day of the measurement, etc. are unavailable for many studies.

- Sample used: some of the present compilation is based on single study, single measurement data, while others have made multiple observations on the chosen subjects. For many of the activities data are not available for both genders.

- It is important to recognise that the PARs presented, represent data related to the actual activity and are not inclusive of the rest periods that may be associated with the normal performance of the activity.

- Inadequate coverage: there are some areas of human activity that are not represented in the final compilation because data was not accessible to the author, or because no data appears to be available. Similarly, nonEnglish data sources have not been reviewed.

\section{Use of the data}

In order to determine the actual energy cost of an activity, it is necessary to first determine the BMR, either by actual measurement or by using prediction equations. The energy cost of the activity is then computed by multiplying the BMR of the individual with the PAR reported in the table. When this is multiplied by the duration (in minutes) of the activity, the total energy expenditure related to the activity is obtained. Thus as an example, if an individual who had a BMR of $1.0 \mathrm{kcal} \mathrm{min}^{-1}$, washes clothes while squatting on the ground ( $\mathrm{PAR}=2.8$ ) for 10 minutes, the total energy expended will be $1.0 \times 2.8 \times 10=28 \mathrm{kcal}$.

There are several important problems that will be faced by people who use the tables.

\section{Does a small difference in PARs between genders imply a significant gender difference in the energy cost of an activity?}

It is important to recognise that the PARs presented in the final compilation are actually computed PARs from the studies that have been reviewed. A small difference in the PAR between the genders may thus be of no significance. Differences between the genders in the final compilation could be attributed to various factors including different sources of data, the number of sources available, and different methods of performing the activity, among others.

\section{PARs are available for only one gender}

In the present compilation, 62 activities were identified for which there were matching data for both males and females from the same study, over a wide PAR range. Male and female PARs were highly correlated $(r=0.97)$. Female PARs could be predicted from the male PAR using the equation $0.968 \times$ Male $\mathrm{PAR}+0.194$. Based on this analysis, it would be reasonable to use the PAR of the available gender for both genders, when data is not available for both males and females.

\section{The PAR for an activity is not listed}

Where the PAR for an activity is not listed, it may be necessary to use the PAR of an activity that is closely related or similar in intensity. While this calls for judgement by the user, there appears to be no alternative for this, until such data are generated.

\section{The average PAR for an activity seems too high or too low}

This is because the average PAR in the final compilation is an average of the PARs obtained from different studies. The value across studies is quite variable and may, in part, be due to the variable way in which the same activity has been performed in the different studies as well as measurement errors. In such a situation, the PAR range has been provided, which is basically the lowest and highest PAR reported across studies. The range will allow the user to make a decision about which value within the range provided is appropriate for the activity, performed under specific conditions.

\section{What PAR should be applied when an activity has been performed for a long duration with obvious rest periods or pauses?}

Since the PARs in the present compilation, like those of the 1985 compilation, reflect values of activities during the actual performance of the activity, application of the PARs for durations when there have been rest periods/pauses will tend to overestimate the energy expenditure. One of the approaches to this problem may be to assign a correction factor that takes into account rest pauses. A case in point, where this has been attempted is James and Schofield $^{3}$, based on the earlier FAO/WHO/UNU Expert Consultation, where the authors have suggested the use of Integrated Energy Indices (IEI), which are essentially PARs, corrected for pauses/rest periods. According to the 
method proposed, PARs can be divided into light, moderate and heavy activities based on PAR cut-offs of $1.0-2.5,2.6-3.9$ and $4.0+$. Average estimates of the length of pauses during specified activities is estimated at $75 \%$ of the time of light activities, $25 \%$ of the time for moderate activities and $40 \%$ of the time for heavy activities. The average estimates of PAR for the rest periods/pauses are 1.54 for males and 1.68 for females.

\section{Conclusion}

The present compilation has been based on the need to provide data on adults for a wide spectrum of human activity. There are, however, lacunae in the available data for many activities, between genders, across age groups and in various physiological states. Future work needs to target these areas, while addressing the limitations of the available data listed earlier.

\section{Acknowledgements}

The authors would like to acknowledge support received from the Nestle Foundation, Switzerland, for the collection of data over a period of nearly 2 years.

\section{References}

1 WHO. Energy and Protein Requirements. Report of a Joint FAO/WHO/UNU Expert Consultation. Technical Report Series No. 724. Geneva: World Health Organization, 1985

2 Passmore R, Durnin JVGA. Human energy expenditure. Physiological Reviews 1955; 35: 801-40.

3 James WPT, Schofield EC. Human Energy Requirements. A Manual for Planners and Nutritionists. Oxford: Oxford Medical Publications, 1990.

4 Almero EM, de Guzman PE, Cabera JP, Yuchingtat GP, Piguing MC, Gaurano AL, Caguiat JO, Zolanzo FG, Alina FT. A study on the metabolic costs of activities and dietary intake of some construction workers. Philippine Journal of Nutrition 1984; 37: 49-56.

5 Bandyopadhyay B, Chattopadhyay H. Energy metabolism in male college students. Indian Journal of Medical Research 1980; 71: 961-9.

6 Banerjee B, Saha N. Resting metabolic rate and energy cost of some common daily activities of trained and untrained tropical people. Journal of Sports Medicine 1972; 12: $111-6$.

7 Barnes RM. Physical energy expenditure in long-haul cabin crew. Aerospace Medicine 1973; 44(7): 783-5.

8 Bleiberg FM, Brun TA, Goihman S. Duration of activities and energy expenditure of female farmers in dry and rainy seasons in Upper Volta. British Journal of Nutrition 1980; 43: $71-82$.

9 Brun TA, Geissler CA, Mirbagheri I, Hormozdiary H, Bastani J, Hedayat $H$. The energy expenditure of Iranian agricultural workers. American Journal of Clinical Nutrition 1979; 32 : 2154-61.

10 Brun T, Bleiberg F, Goihman S. Energy expenditure of male farmers in dry and rainy seasons in Upper Volta. British Journal of Nutrition 1981; 45: 67-75.

11 Brun T. The assessment of total energy expenditure of female farmers under field conditions. Journal of Biosocial Science 1992; 24: 325-33.
12 Cassady S1, Nielsen DH. Cardiorespiratory responses of healthy subjects to calisthenics performed on land versus in water. Physical Therapy 1992; 72: 532-8.

13 Cole AH, Ogbe JO. Energy intake, expenditure and pattern of daily activity of Nigerian male students. British Journal of Nutrition 1987; 58: 357-67.

14 Costa G, Berti F, Betta A. Physiological cost of apple-farming activities. Applied Ergonomics 1989; 20: 281-6.

15 Das SK, Saha H. Climbing efficiency with different modes of load carriage. Indian Journal of Medical Research 1966; 54: 866-71.

16 Datta SR, Chatterjee BB, Roy BN. The energy cost of rickshaw pulling. Ergonomics 1978; 21: 879-86.

17 Datta SR, Chatterjee BB, Roy BN. The energy cost of pulling handcarts ('thela'). Ergonomics 1983; 26: 461-4.

18 Davies CTM, Brotherhood JR, Collins KJ, et al. Energy expenditure and physiological performance of Sudanese cane cutters. British Journal of Industrial Medicine 1976; 33 : $181-6$.

19 de Guzman MPE, Kalaw JM, Tan RH, Recto RC, Basconcillo RO, Ferrer VT, Tumbokon MS, Yuchingtat GP, Gaurano AL. A study of the energy expenditure, dietary intake and pattern of daily activity among various occupational groups. Urban Jeepney Drivers. Philippine Journal of Nutrition 1974; 27 : 182-8.

20 de Guzman PE, Dominguez SR, Kalaw JM, Buning MN, Basconcillo RO, Santos VF. A study of the energy expenditure, dietary intake and pattern of daily activity among various occupational groups. Marikina Shoemakers and housewives. Philippine Journal of Nutrition 1974; 27: $21-30$.

21 de Guzman MPE, Cabrera JP, Basconcillo RO, Gaurano AL, Yuchingtat GP, Tan RM, Kalaw JM, Recto RC. A study of the energy expenditure, dietary intake and pattern of daily activity among various occupational groups. Clerk-typist. Philippine Journal of Nutrition 1978; 31: 147-56.

22 de Guzman Ma PE, Recto Ma RC, Cabera JP, Basconcillo RO, Gaurano AL, Yuchingtat GP, Abanto ZU. A study of the energy expenditure, dietary intake and pattern of daily activity among various occupational groups. Textile Mill workers. Philippine Journal of Nutrition 1979; 32: 134-48.

23 de Guzman Ma PE, Cabera JP, Yuchintat GP, Abanto ZU, Gaurano AL. A study of energy expenditure, dietary intake and pattern of daily activity among various occupational groups. Laguna Rice farmers. Philippine Journal of Nutrition 1984; 37: 163-74.

24 di Prampero PE, Pendergast DR, Wilson DW, Rennie DW. Energetics of swimming in man. Journal of Applied Physiology 1974; 37: 1-5.

25 Dufour DL. The time and energy expenditure of indigenous women horticulturalists in the Northwest Amazon. American Journal of Physical Anthropology 1984; 65: 37-46.

26 Edholm OG, Fletcher JG. The energy expenditure and food intake of individual men. British Journal of Nutrition 1955; 9: 286-300.

27 Edholm OG, Humphrey S, Lourie JA, Tredre BE, Brotherhood J. Energy expenditure and climatic exposure of Yemenite and Kurdish Jews in Israel. Philosophical Transactions of the Royal Society of London. Series B, Biological Sciences 1973; 266: 127-40.

28 Edmundson WC, Edmundson SA. Energy balance, nutrient intake and discretionary activity in a South Indian village. Ecology of Food and Nutrition 1989; 22: 253-65.

29 Fariduddin KM, Mujibur Rahman M, Ahsanullah ABM. Study of energy expenditure and food intake of some working class people of Bangladesh. Bangladesh Medical Research Council Bulletin 1975; 1: 24-31.

30 Fariduddin KM, Mujibur Rahaman M. Study of energy expenditure and food intake of some working class people 
of Bangladesh. Part II. Bangladesh Medical Research Council Bulletin 1976; 2: 27-30.

31 Garby L, Kurzer MS, Lammert O, Nielsen E. Energy expenditure during sleep in men and women: evaporative and sensible heat losses. Human Nutrition: Clinical Nutrition 1987; 41C: 225-33.

32 Haisman MF, Winsmann FR, Goldman RF. Energy cost of pushing loaded handcarts. Journal of Applied Physiology 1972; 33: 181-3.

33 Igbanugo V, Gutin B. The energy cost of aerobic dancing. Operational Research Quarterly 1978; 49: 308-16.

34 Jette M, Mongeon J, Routhier R. The energy cost of rope skipping. Journal of Sports Medicine 1979; 19: 33-7.

35 Jing L, Wenyu Y. The energy expenditure and nutritional status of college students. The energy cost and the total energy expenditure per day. Biomedical and Environmental Sciences 1991; 4: 295-303.

36 Lawrence M, Singh J, Lawrence F, Whitehead RG. The energy cost of common daily activities in African women: increased expenditure in pregnancy. American Journal of Clinical Nutrition 1985; 42: 753-63.

37 Lemon PWR, Hermiston RT. The human energy cost of fire fighting. Journal of Occupational medicine Official Publication of the Industrial Medical Association 1977; 19: 558-62.

38 Littell DE, Joy RJT. Energy cost of piloting fixed- and rotarywing aircraft. Journal of Applied Physiology 1969; 26: 282-5.

39 Louhevaara V, Teraslinna P, Pirila P, Salmio S, Ilmarinen J. Physiological responses during and after intermittent sorting of postal parcels. Ergonomics 1988; 31: 1165-75.

40 Malhotra MS, Chandra U, Sridharan K. Dietary intake and energy requirements of Indian submariners in tropical waters. Ergonomics 1976; 19: 141-8.

41 Marchetti M, Figura F, Ricci B. Biomechanics of two fundamentally sailing postures. Journal of Sports Medicine 1980; 20: 325-32.

42 Montgomery E, Johnson A. Machiguenga energy expenditure. Ecology of Food and Nutrition 1977; 6: 97-105.

43 Nag PK, Dutt P. Circulo-respiratory efficiency in some agricultural work. Applied Ergonomics 1980; 11: 81-4.

44 Nag PK, Chatterjee SK. Physiological reactions of female workers in Indian agricultural work. Human Factors 1981; 23: 607-14.

45 Norgan NG, Ferro-Luzzi A, Durnin JVGA. The energy and nutrient intake and the energy expenditure of 204 New Guinean adults. Philosophical Transactions of the Royal Society of London. Series B, Biological Sciences 1974; 268: 309-48.

46 O'Connell ER, Thomas PC, Cady LD, Krwasky RJ. Energy costs of simulated stair climbing as a job related task in fire fighting. Journal of Occupational medicine Official Publication of the Industrial Medical Association 1986; 28: 282-4.

47 Oberoi K, Dhillon MK, Miglani SS. A study of energy expenditure during manual and machine washing of clothes in India. Ergonomics 1983; 26: 375-8.

48 Pal AK, Sinha DK. The energy cost of metalliferous mining operations in relation to the aerobic capacity of Indian miners. Ergonomics 1994; 37: 1047-54.

49 Phillips PG. The metabolic cost of common West African agricultural activities. Tropical Medicine and Hygiene 1954; 57: 12-20.

50 Raven PB, Colwell MO, Drinkwater BL, Horvath SM. Indirect calorimetric estimation of specific tasks of aluminium smelter workers. Journal of Occupational medicine Official Publication of the Industrial Medical Association 1973; 15: 894-8.

51 Ramana Murthy PSV, Belavady B. Energy expenditure and requirement in agricultural labourers. Indian Journal of Medical Research 1966; 54: 977-9.

52 Samanta A, Chatterjee BB. A physiological study of manual lifting of loads in Indians. Ergonomics 1981; 24: 557-64.

53 Samanta A, Datta SR, Roy BN, Chaterjee A, Mukherjee PK. Estimation of maximal permissible loads to be carried by Indians of different ages. Ergonomics 1987; 30: 825-31.

54 Schmidt RJ, Housh TJ, Hughes RA. Metabolic response to Kendo. Journal of Sports Medicine 1985; 25: 202-6.

55 Sheldahl LM, Wilke NA, Dougherty SM, Levandoski SG, Hoffman MD, Tristani FE. Effect of age and coronary artery disease on response to snow shoveling. Journal of the American College of Cardiology 1992; 20: 1111-7.

56 Spurr GB, Barac-Nieto M, Maksud MG. Energy expenditure cutting sugar cane. Journal of Applied Physiology 1975; 39: 990-6.

57 Spurr GB, Maksud MG, Barac-Nieto M. Energy expenditure, productivity, and physical work capacity of sugar cane loaders. American Journal of Clinical Nutrition 1977; 30: 1740-6.

58 Thornton R, Brown GA, Higenbottam C. The energy expenditure of helicopter pilots. Aviation space and Environmental Medicine 1984; 55: 746-50.

59 Tin-May-Than. Energy expenditure, duration of activities, and physical work capacities of Burmese women weavers. Food and Nutrition Bulletin 1988; 10: 48-50.

60 Torun B, McGuire J, Mendoza RD. Energy cost of activities of women from a rural region of Guatemala. Nutrition Research 1982; 2: 127-36.

61 Town GP, Sol N, Sinning WE. The effect of rope skipping rate on energy expenditure of males and females. Medicine and Science in Sports and Exercise 1980; 12: 295-8.

62 Viteri FE, Turun B, Galicia CJ, Herrera E. Determining energy costs of agricultural activities by respirometer and energy balance techniques. American Journal of Clinical Nutrition 1971; 24: 1418-30.

63 Wilke NA, Sheldahl LM, Dougherty SM, Hanna RD, Nickele GA, Tristani FE. Energy expenditure during household tasks in women with coronary artery disease. American Journal of Cardiology 1995; 75: 670-4.

64 Wilmore J, Parr RB, Ward P, Vodak PA, Barstow TJ, Pipes TV, Grimditch G, Leslie P. Energy cost of circuit weight training. Medicine and Science in Sports 1978; 10: 75-8.

65 Zhuo D, Shephard RJ, Plyley MJ, Davis GM. Cardiorespiratory and metabolic responses during Tai Chi Chuan Exercise. Canadian Journal of Applied Sport Sciences 1984; 9: 7-10.

\section{Appendix}

\section{A compilation of the details of the individual studies used for the collation of data on energy costs of specified activities}

Studies are presented according to alphabetical order of the first author. 
Table 1 Almero, 1984. Energy costs of construction workers in the Philippines ${ }^{4}$

\begin{tabular}{|c|c|c|c|c|}
\hline \multirow[b]{2}{*}{ Activities } & \multicolumn{2}{|c|}{$\begin{array}{c}\text { Energy cost of } \\
\text { activity }\left(\mathrm{kcal} \mathrm{min}^{-1}\right)\end{array}$} & \multicolumn{2}{|c|}{ PAR } \\
\hline & Men & Women & Men & Women \\
\hline \multicolumn{5}{|l|}{ General labour } \\
\hline Carrying box with load of $8-12 \mathrm{~kg}$ & 4.902 & & 4.41 & \\
\hline Mix cement using shovel & 5.866 & & 5.27 & \\
\hline Tapping-chipping cement walls & 3.647 & & 3.28 & \\
\hline Shovel sand & 7.89 & & 7.09 & \\
\hline Sift sand using sieve & 4.712 & & 4.24 & \\
\hline Carry H-blocks & 2.336 & & 2.1 & \\
\hline Acid clean tiles & 3.894 & & 3.5 & \\
\hline Carry-transfer wood & 7.684 & & 6.91 & \\
\hline \multicolumn{5}{|l|}{ Masonry } \\
\hline Smooth surface of cemented walls & 4.940 & & 4.44 & \\
\hline Tapping-chipping tiles & 1.804 & & 1.62 & \\
\hline Grouting joints of tiles and blocks & 3.902 & & 3.51 & \\
\hline Aligning blocks & 5.828 & & 5.24 & \\
\hline Tapping to bore hole on cement walls/floors & 4.083 & & 3.67 & \\
\hline Plastering using wood float & 3.155 & & 2.84 & \\
\hline Make sandblaster & 2.703 & & 2.43 & \\
\hline Sandblast & 5.046 & & 4.54 & \\
\hline Steel brush wash out & 3.820 & & 3.43 & \\
\hline \multicolumn{5}{|l|}{ Carpentry } \\
\hline Sawing wood & 5.596 & & 5.03 & \\
\hline Planing & 6.249 & & 5.62 & \\
\hline Form framework (by sawing and hammering) & 3.995 & & 3.592 & \\
\hline Form parquet (put in place) & 5.793 & & 5.21 & \\
\hline Make cabinet doors and hinges & 3.824 & & 3.44 & \\
\hline Drilling wood & 5.855 & & 5.26 & \\
\hline \multicolumn{5}{|l|}{ Electricals } \\
\hline Hook up wire for PVC & 3.844 & & 3.46 & \\
\hline Tapping-splicing & 3.358 & & 3.02 & \\
\hline \multicolumn{5}{|l|}{ Painting } \\
\hline Painting & 4.012 & & 3.61 & \\
\hline Varnishing & 3.562 & & 3.2 & \\
\hline Sandpaper balustrade & 3.180 & & 2.86 & \\
\hline
\end{tabular}

Abbreviations: BMR - basal metabolic rate; PAR - physical activity ratio.

Subjects: 25 males, age $25 \pm 5$ years, height $161 \pm 6 \mathrm{~cm}$, weight $51 \pm 5 \mathrm{~kg}$ (all mean $\pm \mathrm{SD}$ ).

Equipment: Max Planck respirometer calibrated according to the method of Durnin. Expired $\mathrm{O}_{2}$ measured using Beckman E2 analyser. Measurements: 8 min collections made, 1-2 determinations/activity/subject.

BMR measured in 19 subjects using the Sanborn metabolism apparatus.

PAR calculated using measured BMRs.

Table 2 Bandyopadhyay et al., 1980. Energy cost of some basic activities of college students in India ${ }^{5}$

\begin{tabular}{|c|c|c|c|c|}
\hline \multirow[b]{2}{*}{ Activities } & \multicolumn{2}{|c|}{$\begin{array}{l}\text { Energy cost of } \\
\text { activity }\left(\mathrm{kcal} \mathrm{min} \mathrm{min}^{-1}\right)\end{array}$} & \multicolumn{2}{|c|}{ PAR } \\
\hline & Men & Women & Men & Women \\
\hline Lying resting & 0.93 & & 1.07 & \\
\hline Sitting resting, sitting and eating, sitting on trains, buses etc. & 1.01 & & 1.16 & \\
\hline Standing resting & 1.07 & & 1.23 & \\
\hline Sitting studying & 1.11 & & 1.28 & \\
\hline Sitting working; shoe polishing, washing clothes & 1.18 & & 1.36 & \\
\hline Standing working; bathing, dressing, shaving etc. & 1.43 & & 1.64 & \\
\hline Walking & 2.28 & & 2.62 & \\
\hline
\end{tabular}

Abbreviations: BMR - basal metabolic rate; PAR - physical activity ratio.

Subjects: all male, 9 athletes and 11 non-athletes. Data collation restricted to non-athletes. Age $19 \pm 2.4$ years, height $167.5 \pm 7.4 \mathrm{~cm}$, weight $51.1 \pm 6.7 \mathrm{~kg}$, per cent fat $10.1 \pm 2.2$ (all mean \pm SD).

Equipment: Douglas bag, with $\mathrm{O}_{2}$ estimation from Scholander micrometer.

Measurements: duration of activities not given. BMR for the group $0.87 \pm 0.032 \mathrm{kcal} \mathrm{min}^{-1}$.

PAR calculated using measured BMRs. 
Table 3 Banerjee et al., 1972. Variety of tasks in Singapore ${ }^{6}$

\begin{tabular}{lccccc}
\hline & \multicolumn{2}{c}{$\begin{array}{c}\text { Energy cost of } \\
\text { activity }\left(\mathrm{kcal} \mathrm{min}{ }^{-1}\right)\end{array}$} & & \multicolumn{2}{c}{ PAR } \\
\cline { 2 - 3 } Activities & Men & Women & & Men & Women \\
\hline Sitting & 1.03 & 0.68 & 1.06 & 1.05 \\
Sitting and reading & 1.03 & 0.70 & 1.06 & 1.08 \\
Sitting and writing & 1.11 & 0.78 & & 1.14 & 1.2 \\
Standing & 1.39 & 0.89 & & 1.43 & 1.37 \\
Walking (on a level ground at 4-4.8 $\mathrm{km} \mathrm{h}^{-1}$ ) & 2.98 & 2.10 & & 3.07 & 3.23 \\
Running (on a level ground at 7-9 $\mathrm{km} \mathrm{h}^{-1}$ ) & 6.15 & 4.55 & 6.34 & 7 \\
\hline
\end{tabular}

Abbreviations: BMR - basal metabolic rate; PAR - physical activity ratio.

Subjects: Data are presented here only for the sedentary (untrained) subjects - Ten 18-25-year-old male medical students; height $172.5 \mathrm{~cm}$, weight $65.0 \mathrm{~kg}$, Lean body mass $50.4 \mathrm{~kg}$ and 10 females; height $157.0 \mathrm{~cm}$, weight $47.5 \mathrm{~kg}$, Lean body mass $36.2 \mathrm{~kg}$ (all mean).

Equipment: Max Planck respirometer with Lloyd's gas analysis apparatus for $\mathrm{O}_{2}$ and $\mathrm{CO}_{2}$

Measurements: for 5 minutes. With 10 minute breaks between tests.

PAR calculated using measured BMR $\left(0.97 \mathrm{kcal} \mathrm{min}^{-1}\right.$ for men, $0.65 \mathrm{kcal} \mathrm{min}{ }^{-1}$ for women, mean).

Table 4 Barnes et al., 1973. Energy costs of activities during long haul cabin activities ${ }^{7}$

\begin{tabular}{|c|c|c|c|c|}
\hline \multirow[b]{2}{*}{ Activities } & \multicolumn{2}{|c|}{$\begin{array}{c}\text { Energy cost of } \\
\text { activity }\left(\mathrm{kcal} \mathrm{min}^{-1}\right)\end{array}$} & \multicolumn{2}{|c|}{ PAR } \\
\hline & Men & Women & Men & Women \\
\hline \multicolumn{5}{|l|}{ Galley work } \\
\hline First class & 3.67 & 2.89 & 3.02 & 3.19 \\
\hline Economy: light meal & 3.19 & 2.5 & 2.62 & 2.76 \\
\hline Economy: main meal & 4.12 & 3.24 & 3.69 & 3.58 \\
\hline \multicolumn{5}{|l|}{ Serving meals } \\
\hline First class & 3.26 & 2.56 & 2.68 & 2.83 \\
\hline Economy trays by hand, light meal & 4.2 & 3.3 & 3.46 & 3.64 \\
\hline Economy: trays by hand, main meal & 3.78 & 2.97 & 3.11 & 3.28 \\
\hline Mixed mobile tray box & 3.86 & 3.03 & 3.18 & 3.34 \\
\hline \multicolumn{5}{|l|}{ Bar service } \\
\hline Dispensing & 4.11 & 3.23 & 3.38 & 3.57 \\
\hline Serving & 2.67 & 2.1 & 2.2 & 2.32 \\
\hline Standard walking $\left(3 \mathrm{~m} \mathrm{~h}^{-1}\right)$ & 4.12 & 3.56 & 3.39 & 3.93 \\
\hline
\end{tabular}

Abbreviations: BMR - basal metabolic rate; PAR - physical activity ratio.

Subjects: characteristics not described. Energy costs for a $70 \mathrm{~kg}$ man and $55 \mathrm{~kg}$ woman.

Equipment: Max Planck respirometer with Lloyd Haldane gas analyser.

Measurements: for 10 minutes. Details of gas collection and calibration not given

BMR not measured. PAR based on predicted BMR assuming an age range of $18-30$ years.

Table 5 Bleiberg et al., 1980. Energy expenditure of various farming activities in female Upper-Volta farmers ${ }^{8}$

\begin{tabular}{|c|c|c|c|c|c|}
\hline \multirow[b]{2}{*}{ Activities } & \multirow[b]{2}{*}{$n$} & \multicolumn{2}{|c|}{$\begin{array}{c}\text { Energy cost of } \\
\left.\text { activity (kcal min }{ }^{-1}\right)\end{array}$} & \multicolumn{2}{|c|}{ PAR } \\
\hline & & Men & Women & Men & Women \\
\hline Lying & 29 & & 1.24 & & 1.37 \\
\hline Sitting & 27 & & 1.29 & & 1.42 \\
\hline Standing & 27 & & 1.35 & & 1.49 \\
\hline Walking & 18 & & 3.0 & & 3.31 \\
\hline Sowing & 4 & & 3.9 & & 4.31 \\
\hline Thinning out and replanting & 5 & & 3.6 & & 3.97 \\
\hline Hoeing & 11 & & 4.3 & & 4.75 \\
\hline Grinding grain on a millstone & 24 & & 4.2 & & 4.64 \\
\hline Pounding & 32 & & 4.5 & & 4.97 \\
\hline Fetching water from a well & 21 & & 4.1 & & 4.53 \\
\hline Fetching water from the swamp & 2 & & 3.9 & & 4.31 \\
\hline Washing clothes & 3 & & 3.2 & & 3.53 \\
\hline Stirring sorghum or millet porridge & 7 & & 3.7 & & 4.09 \\
\hline
\end{tabular}

Abbreviations: BMR - basal metabolic rate; PAR - physical activity ratio.

Subjects: numbers variable for each activity. Age 30.6 years, height $157 \mathrm{~cm}$, weight $50.6 \mathrm{~kg}$, per cent fat 19.3 (all means).

Equipment: Kofranyi-Michaelis respirometer. Expired air analysed within 5 minutes of collection with Servomex $\mathrm{O}_{2}$ analyser.

Measurement: for approx 10 minutes for each activity. Energy costs were recalculated for a standard wt of $55 \mathrm{~kg}$.

PAR calculated using BMR predicted for a body wt of $55 \mathrm{~kg}$. 
Table 6 Brun, 1979. Energy cost of Iranian agricultural workers across four seasons ${ }^{9}$

\begin{tabular}{|c|c|c|c|c|}
\hline \multirow[b]{2}{*}{ Activities } & \multicolumn{2}{|c|}{$\begin{array}{l}\text { Energy cost of activity } \\
\quad\left(\mathrm{kcal} \mathrm{h}^{-1} \mathrm{~min}^{-1}\right)\end{array}$} & \multicolumn{2}{|c|}{ PAR } \\
\hline & Men & Women & Men & Women \\
\hline \multicolumn{5}{|l|}{ Cotton harvest } \\
\hline Picking cotton and carrying sack & 3.6 & & 3.21 & \\
\hline Loading, collecting sacks on lorry & 7.1 & & 6.34 & \\
\hline \multicolumn{5}{|l|}{ Irrigation } \\
\hline Opening and closing irrigation channels & 4.5 & & 4.01 & \\
\hline \multicolumn{5}{|l|}{ Digging activities } \\
\hline Channel digging & 7.0 & & 6.25 & \\
\hline Digging & 6.4 & & 5.71 & \\
\hline \multicolumn{5}{|l|}{ Cultivation } \\
\hline Weeding & 5.2 & & 4.64 & \\
\hline Tending melons & & & & \\
\hline \multicolumn{5}{|l|}{ Grain harvest } \\
\hline Tending threshing machine & 3.8 & & 3.39 & \\
\hline Lifting grain sacks (weighing and loading) & 4.0 & & 3.57 & \\
\hline Winnowing & 4.0 & & 3.57 & \\
\hline \multicolumn{5}{|l|}{ Animals } \\
\hline Tending animals & 5.1 & & 4.55 & \\
\hline Collecting and spreading manure & 5.5 & & 4.91 & \\
\hline Loading manure & 6.8 & & 6.07 & \\
\hline \multicolumn{5}{|l|}{ Transport } \\
\hline Riding donkey/tractor & 2.9 & & 2.59 & \\
\hline Cycling on level dirt road & 5.6 & & 5.0 & \\
\hline \multicolumn{5}{|l|}{ Railway work } \\
\hline Raking gravel & 4.7 & & 4.2 & \\
\hline Using pick under the rails & 7.7 & & 6.87 & \\
\hline \multicolumn{5}{|l|}{ Roof repair } \\
\hline Shovelling and carrying mud & 5.9 & & 5.27 & \\
\hline Spreading mud on roof & 2.9 & & 2.59 & \\
\hline
\end{tabular}

Abbreviations: BMR - basal metabolic rate; PAR - physical activity ratio.

Subjects: 45 males, age ?, mean $35-39$ years, mean weight across seasons $56-59 \mathrm{~kg}$.

Equipment: Max Planck respirometer with a Pauling $\mathrm{O}_{2}$ analyser.

Measurements: most activities measured for 10-15 min. No BMR

PAR calculated using predicted BMR (age range 30-60years and an avg wt of $57.5 \mathrm{~kg}$ ) and based on recomputed energy costs expressed in $\mathrm{kcal} \mathrm{min}^{-1}$. 
Table 7 Brun, 1981. Energy cost of a variety of agricultural activities in Upper-Volta farmers ${ }^{10}$

\begin{tabular}{|c|c|c|c|c|c|}
\hline \multirow[b]{2}{*}{ Activities } & \multirow[b]{2}{*}{$n$} & \multicolumn{2}{|c|}{$\begin{array}{l}\text { Energy cost of } \\
\text { activity }\left(\mathrm{kcal} \mathrm{min} \mathrm{min}^{-1}\right)\end{array}$} & \multicolumn{2}{|c|}{ PAR } \\
\hline & & Men & Women & Men & Women \\
\hline Lying & 31 & 1.39 & & 1.25 & \\
\hline Sitting & 33 & 1.38 & & 1.24 & \\
\hline Standing & 29 & 1.44 & & 1.29 & \\
\hline Walking & 25 & 3.6 & & 3.23 & \\
\hline Walking slowly & 4 & 2.9 & & 2.6 & \\
\hline Walking fast & 2 & 4.2 & & 3.77 & \\
\hline Cycling & 12 & 4.4 & & 3.94 & \\
\hline Sowing & 5 & 3.9 & & 3.5 & \\
\hline Thinning out and replanting & 8 & 3.8 & & 3.41 & \\
\hline Hoeing & 11 & 5.1 & & 4.57 & \\
\hline Land clearing & 2 & 6.9 & & 6.19 & \\
\hline $\begin{array}{l}\text { Sorghum harvest: standing, cutting the ears with } \\
\text { knife or hand }\end{array}$ & 6 & 2.4 & & 2.15 & \\
\hline Bent forward, uprooting potatoes with a hoe & 5 & 3.9 & & 3.5 & \\
\hline Plucking leaves and stems, standing & 1 & 6.8 & & 6.1 & \\
\hline Kneeling and sorting, sweet potatoes & 1 & 1.8 & & 1.61 & \\
\hline Cutting straw with a sickle, bent forward & 3 & 5.6 & & 5.02 & \\
\hline Walking with a sheaf of straw on head $(11.5 \mathrm{~kg})$ & 1 & 3.4 & & 3.05 & \\
\hline $\begin{array}{l}\text { Pulling and breaking into pieces branches from } \\
\text { dead trees, walking and bending forward }\end{array}$ & 2 & 3.8 & & 3.41 & \\
\hline Cutting wood with a machete & 1 & 4.6 & & 4.12 & \\
\hline Unloading a cart of branches & 2 & 3.6 & & 3.23 & \\
\hline Vine weaving & 2 & 2.4 & & 2.15 & \\
\hline Hand weaving sitting on the ground & 2 & 2.6 & & 2.33 & \\
\hline Hand sewing & 1 & 1.8 & & 1.61 & \\
\hline Sewing with treadle sewing machine & 3 & 2.4 & & 2.15 & \\
\hline Clay kneading & 1 & 3.0 & & 2.69 & \\
\hline Sawing a calabash by hand, bending forward & 1 & 3.1 & & 2.78 & \\
\hline Making mud bricks squatting & 3 & 3.3 & & 2.96 & \\
\hline Standing, making a mud wall & 1 & 1.8 & & 1.61 & \\
\hline Digging the earth with a pick-axe to make mud & 2 & 6.4 & & 5.74 & \\
\hline Shovelling the mud & 2 & 4.9 & & 4.39 & \\
\hline Copying verses of the Koran, sitting & 1 & 1.2 & & 1.08 & \\
\hline
\end{tabular}

Abbreviations: BMR - basal metabolic rate; PAR - physical activity ratio.

Subjects: all males. Number per activity is variable. Age 32.2 year, height $170 \mathrm{~cm}$, weight $58.5 \mathrm{~kg}$, fat 10.3 (all means).

Equipment: Kofranyi-Michaelis respirometer, expired air analysed with a Servomex $\mathrm{O}_{2}$ analyser.

Measurements: no BMR, all energy costs expressed for a standard weight of $60 \mathrm{~kg}$

PAR based on predicted BMR assuming a wt of $60 \mathrm{~kg}$.

Table 8 Brun, 1992. Not a primary source but reviews data of the author of agricultural activities of Chinese female farmers from Hainan ${ }^{11}$

\begin{tabular}{|c|c|c|c|c|c|}
\hline \multirow[b]{2}{*}{ Activities } & \multirow[b]{2}{*}{$n$} & \multicolumn{2}{|c|}{$\begin{array}{c}\text { Energy cost of } \\
\text { activity }\left(\mathrm{kcal} \mathrm{min}{ }^{-1}\right)\end{array}$} & \multicolumn{2}{|c|}{ PAR } \\
\hline & & Men & Women & Men & Women \\
\hline Sitting inactive & 11 & & 1.08 & & 1.32 \\
\hline Standing resting & 4 & & 1.43 & & 1.74 \\
\hline Squatting washing clothes & 4 & & 2.09 & & 2.55 \\
\hline Standing hoeing & 3 & & 3.82 & & 4.66 \\
\hline Bending, planting potatoes & 7 & & 3.39 & & 4.13 \\
\hline Bending harvesting potatoes & 8 & & 2.36 & & 2.88 \\
\hline Ploughing with buffalo & 4 & & 2.94 & & 3.59 \\
\hline Standing sowing rice & 10 & & 2.15 & & 2.62 \\
\hline Bending, transplanting rice & 31 & & 2.84 & & 3.46 \\
\hline Bending, cutting rice & 26 & & 3.22 & & 3.93 \\
\hline Squatting, bundling rice & 6 & & 2.42 & & 2.95 \\
\hline Standing, threshing rice & 8 & & 3.97 & & 4.84 \\
\hline Walking, carrying $30-35 \mathrm{~kg}$ & 5 & & 3.75 & & 4.57 \\
\hline Walking, tapping rubber & 5 & & 2.52 & & 3.07 \\
\hline
\end{tabular}

Abbreviations: BMR - basal metabolic rate; PAR - physical activity ratio.

Subjects: all women, age ?, average body weight $=47 \mathrm{~kg}$, numbers variable for each activity.

Measurements: for each activity, one or several measurements using Douglas bags or portable spirometers.

PAR calculated using predicted BMR for $18-30$ years and a wt of $47 \mathrm{~kg}$. 
Table 9 Cassady, 1992. Energy cost of calisthenics performed on land and in water ${ }^{12}$

\begin{tabular}{|c|c|c|c|c|}
\hline \multirow[b]{2}{*}{ Activities } & \multicolumn{2}{|c|}{$\begin{array}{c}\text { Energy cost of } \\
\text { activity }\left(\mathrm{VO}_{2} \text { in } 1 \mathrm{~min}^{-1}\right)\end{array}$} & \multicolumn{2}{|c|}{ PAR } \\
\hline & Men & Women & Men & Women \\
\hline Land, upper extremity $20{\text { rep } \min ^{-1}}^{-1}$ & 0.660 & 0.481 & 2.84 & 2.65 \\
\hline Land, lower extremity $20{\text { rep } \min ^{-1}}^{-1}$ & 1.183 & 0.905 & 5.09 & 4.99 \\
\hline Water, upper extremity $20{\text { rep } \min ^{-1}}^{-1}$ & 1.092 & 0.655 & 4.7 & 3.61 \\
\hline 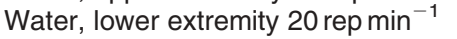 & 1.547 & 1.078 & 6.66 & 5.95 \\
\hline
\end{tabular}

Abbreviations: BMR - basal metabolic rate; PAR - physical activity ratio.

Subjects: 40 healthy subjects (20 men and 20 women), mean age 25 years, mean wt $69.6 \mathrm{~kg}$.

Equipment: open-circuit system. Mouthpiece to Collins gasometer, with Beckman's $\mathrm{O}_{2}$ and $\mathrm{CO}_{2}$ analysers.

Measurements: $\mathrm{O}_{2}$ measurements were made in the final 30 seconds of each exercise stage ( 3 minutes). For the water exercises, the subject performed the exercises in a Hubbard tank with water adjusted to shoulder level for each subject. No BMR.

Energy costs are recalculated from the MET $\left(3.5 \mathrm{ml} \mathrm{kg}^{-1} \mathrm{~min}^{-1}\right)$ and expressed for a standard $65 \mathrm{~kg}$ male and a $55 \mathrm{~kg}$ female.

PAR calculated using predicted BMR.

Table 10 Cole, 1987. Variety of basic activities carried out by Nigerian University students ${ }^{13}$

\begin{tabular}{|c|c|c|c|c|c|}
\hline \multirow[b]{2}{*}{ Activities } & \multirow[b]{2}{*}{$n$} & \multicolumn{2}{|c|}{$\begin{array}{l}\text { Energy cost of } \\
\text { activity }\left(\mathrm{kcal} \mathrm{min}^{-1}\right)\end{array}$} & \multicolumn{2}{|c|}{ PAR } \\
\hline & & Men & Women & Men & Women \\
\hline Sitting & 10 & 1.74 & & 1.18 & \\
\hline Walking at normal pace & 12 & 4.21 & & 2.86 & \\
\hline Personal domestic necessities & 7 & 3.61 & & 2.46 & \\
\hline \multicolumn{6}{|c|}{$\begin{array}{l}\text { Polishing shoes, washing clothes, cleaning } \\
\text { the room, fetching water etc. }\end{array}$} \\
\hline Climbing stairs & 5 & 7.29 & & 4.95 & \\
\hline Lying down in bed awake & 6 & 1.49 & & 1.01 & \\
\hline Standing & 7 & 3.61 & & 2.46 & \\
\hline
\end{tabular}

Abbreviations: BMR - basal metabolic rate; PAR - physical activity ratio.

Subjects: 20 males, numbers different for different activities. Age 24 years, height $171 \mathrm{~cm}$, weight $61.1 \mathrm{~kg}$ (all mean).

Equipment: Max Planck respiration-gasometer.

Measurement: for $10-15$ minutes for each activity.

PAR based on predicted BMR.

Table 11 Costa, 1989. Apple farming activities in Italy ${ }^{14}$

\begin{tabular}{|c|c|c|c|c|}
\hline \multirow[b]{2}{*}{ Activities } & \multicolumn{2}{|c|}{$\begin{array}{l}\text { Energy cost of } \\
\text { activity }\left(\mathrm{kcal} \mathrm{min}^{-1}\right)\end{array}$} & \multicolumn{2}{|c|}{ PAR } \\
\hline & Men & Women & Men & Women \\
\hline $\begin{array}{l}\text { Pruning: done by hand with scissors }(300 \mathrm{~g}) \text { and saw }(500 \mathrm{~g}) \text { standing on } \\
\text { the ground or on a wooden ladder against a tree }\end{array}$ & 4.59 & & 3.6 & \\
\hline $\begin{array}{l}\text { Weeding: cutting and bundling fallen branches using a hatchet }(1900 \mathrm{~g}) \text {, } \\
\text { bill-hook }(700 \mathrm{~g}) \text {, rake }(1000 \mathrm{~g}) \text { and hay-fork }(1600 \mathrm{~g})\end{array}$ & 6.02 & & 4.73 & \\
\hline $\begin{array}{l}\text { Hand spray: of pesticides - hauling a spear }(1000 \mathrm{~g}) \text { connected to a tank } \\
\text { through a flexible rubber tube }\end{array}$ & 4.85 & & 3.81 & \\
\hline $\begin{array}{l}\text { Mech spray: of pesticides - driving a tractor slowly }\left(2-3 \mathrm{~km} \mathrm{~h}^{-1}\right) \text { among } \\
\text { the trees towing a tank provided with an atomiser }\end{array}$ & 2.37 & & 1.86 & \\
\hline $\begin{array}{l}\text { Mowing: cutting the grass among the trees while walking and directing a } \\
\text { self-propelled motor mowing machine }(100 \mathrm{~kg}, 9 \mathrm{hp})\end{array}$ & 6.25 & & 4.91 & \\
\hline $\begin{array}{l}\text { Picking: handling a basket in one hand and picking with the other, } \\
\text { standing on the ground or on a ladder. The full basket can reach } \\
\text { a weight of } 12 \mathrm{~kg}\end{array}$ & 4.58 & & 3.6 & \\
\hline
\end{tabular}

Abbreviations: BMR - basal metabolic rate; PAR - physical activity ratio.

Subjects: 17 males. Age 40.1 years, height 175.4 , weight $80.1 \mathrm{~kg}$ (all means).

Measurement: $\mathrm{VO}_{2}$ measured for short periods (5-10 minutes) by Oxylog in steady state conditions $(<2.5 \%$ incr $)$.

PAR calculated using the predicted BMR. 
Table 12 Das, 1966. Energy cost of different types of load carrying in Indians ${ }^{15}$

\begin{tabular}{|c|c|c|c|c|}
\hline \multirow[b]{2}{*}{ Activities } & \multicolumn{2}{|c|}{$\begin{array}{c}\text { Energy cost of } \\
\text { activity }\left(\mathrm{kcal} \mathrm{min}^{-1}\right)\end{array}$} & \multicolumn{2}{|c|}{ PAR } \\
\hline & Men & Women & Men & Women \\
\hline \multicolumn{5}{|c|}{ Carrying load with shoulder straps } \\
\hline 0 gradient & 2.51 & & 2.27 & \\
\hline $10 \%$ gradient & 5.6 & & 5.05 & \\
\hline $20 \%$ gradient & 8.5 & & 7.67 & \\
\hline \multicolumn{5}{|c|}{ Carrying load with forehead strap } \\
\hline 0 gradient & 2.61 & & 2.43 & \\
\hline $10 \%$ gradient & 6.1 & & 5.5 & \\
\hline $20 \%$ gradient & 8.9 & & 8.03 & \\
\hline \multicolumn{5}{|c|}{ Carrying load on head } \\
\hline 0 gradient & 2.61 & & 2.36 & \\
\hline $10 \%$ gradient & 7.4 & & 6.68 & \\
\hline $20 \%$ gradient & 10.3 & & 9.29 & \\
\hline
\end{tabular}

Abbreviations: BMR - basal metabolic rate; PAR - physical activity ratio. Subjects: six healthy load carriers from the hills. Age 24 years, height $167.8 \mathrm{~cm}$, weight $60 \mathrm{~kg}$

Equipment: Max Planck respirometer with gas analysis on a Scholander's gas analyser.

Measurements: for 5 minutes with gas collections in the last 2 minutes. Load was standard at $27 \mathrm{~kg}$. Speed of treadmill was $3.22 \mathrm{~km} \mathrm{~h}^{-1}$.

PAR calculated using predicted BMR for the group.

Table 13 Datta, 1978. The energy cost of rickshaw pulling by Indian males with different loads ${ }^{16}$

\begin{tabular}{|c|c|c|c|c|c|}
\hline \multirow[b]{2}{*}{ Activities } & \multirow[b]{2}{*}{$n$} & \multicolumn{2}{|c|}{$\begin{array}{l}\text { Energy cost } \\
\text { of activity } \\
(\text { kcal min }\end{array}$} & \multicolumn{2}{|c|}{ PAR } \\
\hline & & Men & Women & Men & Women \\
\hline Rest & 9 & 1.26 & & & \\
\hline $\begin{array}{l}\text { Pulling rickshaw with } \\
\text { no load }\end{array}$ & 10 & 5.39 & & 4.03 & \\
\hline $\begin{array}{l}\text { Pulling rickshaw with } 1 \text { man } \\
\qquad(50+80 \mathrm{~kg})\end{array}$ & 10 & 7.16 & & 5.34 & \\
\hline $\begin{array}{l}\text { Pulling rickshaw with } 2 \text { men } \\
\qquad(100+80 \mathrm{~kg})\end{array}$ & 10 & 8.92 & & 6.66 & \\
\hline $\begin{array}{l}\text { Pulling rickshaw with } 2 \text { men } \\
\text { and } 50 \mathrm{~kg} \text { load } \\
(100+80+50)\end{array}$ & 10 & 11.57 & & 8.64 & \\
\hline
\end{tabular}

Abbreviations: BMR - basal metabolic rate; PAR - physical activity ratio. Subjects: $n=10$. Age 33.8 years, height $162.1 \mathrm{~cm}$, weight $47.8 \mathrm{~kg}$ (all means). Equipment: Douglas bag with Haldane gas analyser.

Measurement: each activity for 10 minutes. Gas collection from 8 minutes to 10 minutes.

PAR calculated using the predicted BMR of the group.

Table 14 Datta, 1983. Energy cost of pulling handcarts in India ${ }^{17}$

\begin{tabular}{|c|c|c|c|c|}
\hline \multirow[b]{2}{*}{ Activities } & \multicolumn{2}{|c|}{$\begin{array}{l}\text { Energy cost } \\
\text { of activity } \\
\left(\text { kcal min }^{-1}\right)\end{array}$} & \multicolumn{2}{|c|}{ PAR } \\
\hline & Men & Women & Men & Women \\
\hline $\begin{array}{l}\text { Rest (10 am 3-4 hours } \\
\text { after morning meal) }\end{array}$ & 1.45 & & 1.1 & \\
\hline No load (190 kg) & 6.34 & & 4.82 & \\
\hline$+185 \mathrm{~kg}$ load & 9.23 & & 7.01 & \\
\hline$+370 \mathrm{~kg}$ load & 12.7 & & 9.64 & \\
\hline
\end{tabular}

Abbreviations: BMR - basal metabolic rate; PAR - physical activity ratio. Subjects: 10 male handcart-pullers. Age 29.6 years, height $165 \mathrm{~cm}$, weight $50 \mathrm{~kg}$ (all means).

Equipment: expired air collected in a Douglas bag and analysed using a Haldane gas analyser.

Measurements: all done at a constant speed of $5 \mathrm{~km} \mathrm{~h}^{-1}$

PAR calculated from the predicted BMR.
Table 15 Davies, 1976. Energy cost of cutting sugar cane in Sudanese $^{18}$

\begin{tabular}{llllll}
\hline & \multicolumn{1}{c}{$\begin{array}{c}\text { Energy cost } \\
\text { of activity } \\
\left(\text { kcal min }^{-1}\right)\end{array}$} & & & \\
\cline { 2 - 3 } \cline { 5 - 6 } Activities & Men $\quad$ Women & & Men & Women \\
\hline $\begin{array}{l}\text { Cutting cane (rate was } \\
\left.15.18 \text { kg min }^{-1}\right)\end{array}$ & 10.96 & & 7.92 & \\
\hline
\end{tabular}

Abbreviations: BMR - basal metabolic rate; PAR - physical activity ratio. Subjects: 42 male cane cutters. Age 26.3 years, height $173.2 \mathrm{~cm}$, weight $54.8 \mathrm{~kg}$ (all means).

Kofranyi-Michaelis respirometer over $20 \mathrm{~min} . \mathrm{O}_{2}$ content with a paramagnetic $\mathrm{O}_{2}$ analyser - Servomex.

PAR calculated based on predicted BMR.

Table 16 de Guzman, 1974. Energy costs of 'Jeepney drivers' in the Philippines ${ }^{19}$

\begin{tabular}{|c|c|c|c|}
\hline \multirow[b]{2}{*}{ Activities } & \multicolumn{2}{|c|}{$\begin{array}{c}\text { Energy } \\
\text { cost of activity } \\
\left(\mathrm{kcal} \mathrm{kg}^{-1} \min ^{-1}\right)\end{array}$} & \multirow{2}{*}{$\begin{array}{l}\text { PAR } \\
\text { Men }\end{array}$} \\
\hline & Men & Women & \\
\hline Driving & 0.046 & & 2.35 \\
\hline Adjust tyres (sitting) & 0.033 & & 1.69 \\
\hline Wipe or clean jeep & 0.038 & & 1.94 \\
\hline Jack up Jeep & 0.067 & & 3.42 \\
\hline Wash Jeep & 0.41 & & 2.09 \\
\hline
\end{tabular}

Abbreviations: BMR - basal metabolic rate; PAR - physical activity ratio. Subjects: 10 males, age 32 years, height $162.2 \mathrm{~cm}$, weight $54.8 \mathrm{~kg}$ (all means).

Equipment: Max Planck respirometer. Gas analysis with E2 Beckman $\mathrm{O}_{2}$ analyser.

Measurements: Basic activities were measured for 10 minutes, others for 8 minutes; $2-3$ determinations of an activity were made on each individual. PAR calculated on the predicted BMR.

Table 17 de Guzman, 1974. Energy cost of activities in Filipino shoemakers and housewives 20

\begin{tabular}{|c|c|c|c|c|}
\hline \multirow[b]{2}{*}{ Activities } & \multicolumn{2}{|c|}{$\begin{array}{c}\text { Energy cost } \\
\text { of activity } \\
\left(\mathrm{kcal} \mathrm{kg}^{-1} \mathrm{~min}^{-1}\right)\end{array}$} & \multicolumn{2}{|c|}{ PAR } \\
\hline & Men & Women & Men & Women \\
\hline \multicolumn{5}{|l|}{ Shoemaker } \\
\hline $\begin{array}{l}\text { Moulding top portion } \\
\text { of shoes }\end{array}$ & 0.054 & & 2.85 & \\
\hline Attaching sole & 0.054 & & 2.85 & \\
\hline Trimming sole and heel & 0.63 & & 3.33 & \\
\hline $\begin{array}{l}\text { Uppermaking (prep of } \\
\text { top portion of shoes) }\end{array}$ & 0.037 & & 2.22 & \\
\hline \multicolumn{5}{|l|}{ Housewife } \\
\hline Bathing child (standing) & & 0.058 & & 3.48 \\
\hline Carrying child (standing) & & 0.032 & & 1.92 \\
\hline Washing clothes & & 0.044 & & 2.64 \\
\hline Cooking & & 0.036 & & 2.16 \\
\hline
\end{tabular}

Abbreviations: BMR - basal metabolic rate; PAR - physical activity ratio. Subjects: eight shoemakers (? male) and 10 housewives (age 34 years, height $155 \mathrm{~cm}$, weight $54 \mathrm{~kg}$, all means).

Equipment: Max Planck respirometer. $\mathrm{O}_{2}$ analysed using Beckman E2 analyser.

Measurements: for each activity for 8 minutes.

PAR calculated using the predicted BMR. 
Table 18 de Guzman, 1978. Energy cost of Filipino clerk-typists ${ }^{21}$

\begin{tabular}{|c|c|c|c|c|c|}
\hline \multirow[b]{2}{*}{ Activities } & \multirow[b]{2}{*}{$n$} & \multicolumn{2}{|c|}{$\begin{array}{l}\text { Energy cost of } \\
\text { activity }\left(\mathrm{kcal} \mathrm{kg}^{-1} \mathrm{~min}^{-1}\right)\end{array}$} & \multicolumn{2}{|c|}{ PAR } \\
\hline & & Men & Women & Men & Women \\
\hline Sitting & & 0.026 & & 1.31 & \\
\hline Standing & & 0.029 & & 1.46 & \\
\hline Walking & & 0.056 & & 2.8 & \\
\hline \multicolumn{6}{|l|}{ Sitting activities } \\
\hline Typing & 10 & 0.035 & & 1.76 & \\
\hline Writing & 4 & 0.028 & & 1.4 & \\
\hline Calculating - desk type electronic calculator & 2 & 0.032 & & 1.61 & \\
\hline Stamping & 1 & 0.023 & & 1.15 & \\
\hline Segregating reports & 1 & 0.023 & & 1.15 & \\
\hline Filing & 2 & 0.026 & & 1.3 & \\
\hline Sorting & 2 & 0.023 & & 1.15 & \\
\hline Proof reading & 1 & 0.023 & & 1.15 & \\
\hline Checking files & 1 & 0.028 & & 1.4 & \\
\hline Checking purchase orders & 1 & 0.028 & & 1.4 & \\
\hline Stapling papers & 1 & 0.028 & & 1.4 & \\
\hline Post-checking & 1 & 0.033 & & 1.66 & \\
\hline Reading & 9 & 0.026 & & 1.3 & \\
\hline Sitting & & & 0.026 & & 1.39 \\
\hline Standing & & & 0.027 & & 1.43 \\
\hline Walking & & & 0.055 & & 2.88 \\
\hline \multicolumn{6}{|l|}{ Sitting activities } \\
\hline Typing & 10 & & 0.035 & & 1.84 \\
\hline Writing & 9 & & 0.028 & & 1.47 \\
\hline Filing & 6 & & 0.028 & & 1.47 \\
\hline Reading & 9 & & 0.028 & & 1.47 \\
\hline Tape recording & 1 & & 0.027 & & 1.42 \\
\hline $\begin{array}{l}\text { Recording transactions } \\
\text { (handwriting) }\end{array}$ & 2 & & 0.026 & & 1.36 \\
\hline $\begin{array}{l}\text { Counting supplies in the stock room, } \\
\text { no lifting involved }\end{array}$ & 1 & & 0.029 & & 1.52 \\
\hline Classifying papers & 1 & & 0.025 & & 1.31 \\
\hline Controlling vouchers & & & 0.025 & & 1.31 \\
\hline Phoning & 1 & & 0.027 & & 1.27 \\
\hline Thermopherring & 1 & & 0.031 & & 1.63 \\
\hline
\end{tabular}

Abbreviations: BMR - basal metabolic rate; PAR - physical activity ratio.

Subjects: 10 males (age 27.6 years, height $165.4 \mathrm{~cm}$, weight $53.9 \mathrm{~kg}$ ) 10 women (age 24.7 years, height $154.8 \mathrm{~cm}$, weight $46.9 \mathrm{~kg}$, all means) Filipino men and women.

Equipment: energy cost using Max Planck respirometer and E2 Beckman analyser.

Measurements: each acivity for 8-10 minutes, 2-3 determinations done per individual.

BMR for each subject on 2 consecutive days using the Sanborn Basal Metabolator - PAR calculated using measured BMR. 
Table 19 de Guzman, 1979. Male and female Filipino textile workers ${ }^{22}$

\begin{tabular}{|c|c|c|c|c|}
\hline \multirow[b]{2}{*}{ Activities } & \multicolumn{2}{|c|}{$\begin{array}{l}\text { Energy cost } \\
\text { of activity } \\
\left(\mathrm{kcal} \mathrm{kg}^{-1} \mathrm{~min}^{-1}\right)\end{array}$} & \multicolumn{2}{|c|}{ PAR } \\
\hline & Men & Women & Men & Women \\
\hline Sitting & 0.022 & & 1.18 & \\
\hline Standing & 0.024 & & 1.26 & \\
\hline Walking & 0.059 & & 3.11 & \\
\hline \multicolumn{5}{|l|}{$\begin{array}{l}\text { Spinning operations } \\
\text { (Operator tends to spinning frames, creels in new roving bobbins during } \\
\text { runouts, pieces-up ends of broken yarns and cleans the machine) }\end{array}$} \\
\hline $\begin{array}{l}\text { Ringframe spinning } \\
\text { Delivering and collecting bobbins and boxes }\end{array}$ & 0.047 & & 2.45 & \\
\hline $\begin{array}{l}\text { (Carriers push loads of boxes of bobbins and deliver them to the } \\
\text { spinners. They also collect used bobbins for cleaning) } \\
\text { Pinwinding }\end{array}$ & 0.095 & & 4.94 & \\
\hline $\begin{array}{l}\text { (Operator rewinds yarns into bobbins used in the weaving shuttles) } \\
\text { Conewinding }\end{array}$ & 0.060 & & 3.12 & \\
\hline $\begin{array}{l}\text { (Operator feeds bobbins into the machine to spin yarns or threads } \\
\text { around big paper cones) }\end{array}$ & 0.065 & & 3.38 & \\
\hline \multicolumn{5}{|l|}{ Weaving operations } \\
\hline Warping & & & & \\
\hline $\begin{array}{l}\text { (Sufficient number of parallel threads are drawn-out from several cones } \\
\text { on creeling stands and rolled into warp beams) } \\
\text { Loading of warp beam }\end{array}$ & 0.059 & & 3.07 & \\
\hline $\begin{array}{l}\text { (Warp beams are loaded into the creeling machine by means of chains } \\
\text { and pulleys) }\end{array}$ & 0.107 & & 5.57 & \\
\hline \multicolumn{5}{|l|}{ Counting yarns per dent } \\
\hline (The threads are sized and counted in preparation for reaching-in) & 0.044 & & 2.29 & \\
\hline Creeling & 0.063 & & 3.28 & \\
\hline Weaving & & & & \\
\hline $\begin{array}{l}\text { (Includes manual movements of cleaning the looms, attaching the loom } \\
\text { beams, feeding bobbins into shuttles, and shuttles into looms, stopping } \\
\text { the machine and piecing up broken ends or changing empty bobbins) } \\
\text { Cloth cutting }\end{array}$ & 0.065 & & 3.38 & \\
\hline $\begin{array}{l}\text { (Finished and filled up loom beams are severed and collected by the } \\
\text { cloth cutter) }\end{array}$ & 0.076 & & 3.96 & \\
\hline Writing (sitting activity) & & & & \\
\hline (Production volume is recorded) & 0.024 & & 1.25 & \\
\hline \multicolumn{5}{|l|}{ Finishing operations } \\
\hline Washing-padding & & & & \\
\hline $\begin{array}{l}\text { (The fabric or woven cloth is brought to the finishing section where it is } \\
\text { washed and de-sized, bleached and printed) }\end{array}$ & 0.044 & & 2.29 & \\
\hline Releasing and dye mixing & 0.048 & & 2.5 & \\
\hline Gig dyeing & 0.049 & & 2.55 & \\
\hline Backtending or high-curing & 0.031 & & 1.61 & \\
\hline Cloth inspecting & 0.024 & & 1.25 & \\
\hline Sitting & & 0.024 & & 1.35 \\
\hline Standing & & 0.024 & & 1.35 \\
\hline Walking & & 0.053 & & 2.95 \\
\hline \multicolumn{5}{|l|}{ Spinning operations } \\
\hline Ringframe spinning & & 0.040 & & 2.24 \\
\hline Conewinding & & 0.040 & & 2.24 \\
\hline \multicolumn{5}{|l|}{ Weaving operations } \\
\hline Warping & & 0.030 & & 1.68 \\
\hline Denting (standing reaching-in) & & 0.050 & & 2.8 \\
\hline Reaching-in (sitting) & & 0.030 & & 1.68 \\
\hline Weaving & & 0.040 & & 2.24 \\
\hline
\end{tabular}

Abbreviations: BMR - basal metabolic rate; PAR - physical activity ratio.

Subjects: Males = 25 (age 24.9 years, height $166 \mathrm{~cm}$, weight $54.8 \mathrm{~kg}$ ), Females = 14 (age 33.1 years, height $153.7 \mathrm{~cm}$, weight $48.7 \mathrm{~kg}$, all means). Variable numbers for different activities.

Equipment: Max Planck respirometer.

Measurements: 10 minutes recording for sitting, standing and walking at own pace; 8 minutes for others; $2-3$ determinations per activity per person.

PAR determined using predicted BMR. 
Table 20 de Guzman, 1984. Agricultural activities of Filipino Laguna rice farmers ${ }^{23}$

\begin{tabular}{|c|c|c|c|c|c|}
\hline \multirow[b]{2}{*}{ Activities } & \multirow[b]{2}{*}{$n$} & \multicolumn{2}{|c|}{$\begin{array}{c}\text { Energy cost of } \\
\text { activity }\left(\mathrm{kcal} \mathrm{kg}^{-1} \mathrm{~min}^{-1}\right)\end{array}$} & \multicolumn{2}{|c|}{ PAR } \\
\hline & & Men & Women & Men & Women \\
\hline Sitting & 9 & 0.027 & & 1.4 & \\
\hline Standing & 9 & 0.027 & & 1.42 & \\
\hline Walking & 9 & 0.061 & & 3.17 & \\
\hline Weeding by hand & 9 & 0.075 & & 3.91 & \\
\hline Mechanical weeding & 4 & 0.123 & & 6.41 & \\
\hline Pushing hand tractor & 9 & 0.119 & & 6.2 & \\
\hline Harvesting & 8 & 0.080 & & 4.17 & \\
\hline Threshing & 7 & 0.115 & & 5.99 & \\
\hline Winnowing & 7 & 0.044 & & 2.29 & \\
\hline Plowing & 9 & 0.126 & & 6.56 & \\
\hline Harrowing & 9 & 0.126 & & 6.56 & \\
\hline Spray & 4 & 0.099 & & 5.16 & \\
\hline Measuring harvested 'palay' & 2 & 0.127 & & 6.61 & \\
\hline Germinating 'palay' & 2 & 0.083 & & 4.32 & \\
\hline Carrying and stacking 'palay' & 2 & 0.100 & & 5.2 & \\
\hline Application of fertiliser & 2 & 0.060 & & 3.13 & \\
\hline Planting & 1 & 0.076 & & 3.96 & \\
\hline Mowing with a scythe & 8 & 0.085 & & 4.43 & \\
\hline Carry 'palay' & 2 & 0.100 & & 5.21 & \\
\hline Sitting & 9 & & 0.027 & & 1.46 \\
\hline Standing & 9 & & 0.029 & & 1.59 \\
\hline Walking & 9 & & 0.050 & & 2.79 \\
\hline Weeding & 9 & & 0.081 & & 4.43 \\
\hline Harvesting & 9 & & 0.080 & & 4.38 \\
\hline Threshing & 7 & & 0.098 & & 5.36 \\
\hline Winnowing & 8 & & 0.053 & & 2.9 \\
\hline Planting & 9 & & 0.085 & & 4.65 \\
\hline
\end{tabular}

Abbreviations: BMR - basal metabolic rate; PAR - physical activity ratio.

Subjects: 10 men (age 27 years, height $159 \mathrm{~cm}$, weight $55 \mathrm{~kg}$ ) 10 women (age 38 years, height $148 \mathrm{~cm}$, weight $47 \mathrm{~kg}$, all means). Variable numbers for different activities.

Equipment: Kofranyi Michaelis respirometer $-\mathrm{O}_{2}$ measured using Beckman analyser.

Measurements: for 8-10 minutes, 2 determinations per person.

PAR calculated using predicted BMR.

Table 21 di Prampero, 1974. Swimming overhead crawl in a $60 \mathrm{~m}$ circum. Annular pool ${ }^{24}$

\begin{tabular}{llllll}
\hline & \multicolumn{2}{c}{$\begin{array}{c}\text { Energy cost of } \\
\text { Activity }\left(\mathrm{kcal} \mathrm{min}{ }^{-1}\right)\end{array}$} & & \multicolumn{2}{c}{ PAR } \\
\cline { 2 - 3 } \cline { 5 - 6 } Activities & Men & Women & & Men & Women \\
\hline Overhead crawl at a velocity of $0.55 \mathrm{~m} \mathrm{~s}^{-1}$ & 11.0 & & 8.45 &
\end{tabular}

Abbreviations: BMR - basal metabolic rate; PAR - physical activity ratio.

Subjects: 10 well-trained male college students, height $175.4 \mathrm{~cm}$, weight $78.2 \mathrm{~kg}$.

Measurements: collection from 4 minutes to 6 minutes of exercise in Douglas Bags. $\mathrm{O}_{2}$ and $\mathrm{CO}_{2}$ analysed by paramagnetic and infrared methods.

PAR calculated using predicted BMR. 
Table 22 Dufour, 1984. Energy costs of mainly horticultural activities of indigenous women in the Amazon ${ }^{25}$

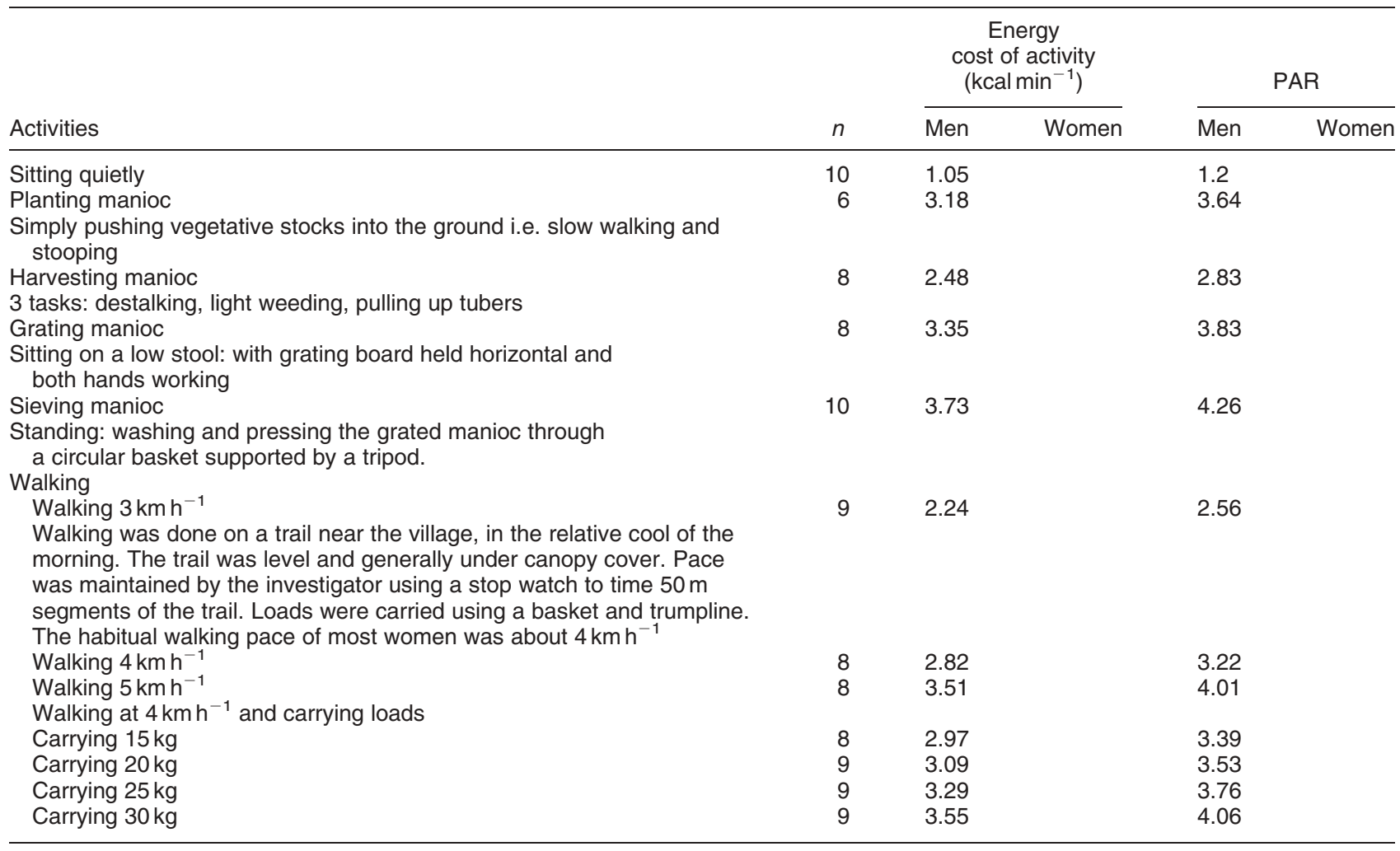

Abbreviations: BMR - basal metabolic rate; PAR - physical activity ratio.

Subjects: Tukanoan Indians, $n=11$, Most non-pregnant and non-lactating (age 34 years, height $147.3 \mathrm{~cm}$, weight $49.6 \mathrm{~kg}$, per cent fat 27 , all means).

Equipment: Kofranyi-Michaelis respirometer. Gas analysis with a Lloyd-Haldane gas analyser.

Measurements: collections for 5-10 minutes following a 5-10 minute warm-up period. Variable number of subjects for different activities.

PAR calculated using predicted BMR.

Table 23 Edholm, 1955. Activities of army cadets ${ }^{26}$

\begin{tabular}{|c|c|c|c|c|c|}
\hline \multirow[b]{2}{*}{ Activities } & \multirow[b]{2}{*}{$n$} & \multicolumn{2}{|c|}{$\begin{array}{l}\text { Energy cost of } \\
\text { activity }\left(\mathrm{kcal} \mathrm{min}^{-1}\right)\end{array}$} & \multicolumn{2}{|c|}{ PAR } \\
\hline & & Men & Women & Men & Women \\
\hline Lying & 12 & 1.48 & & 1.21 & \\
\hline Sitting & 12 & 1.60 & & 1.31 & \\
\hline Standing & 12 & 1.82 & & 1.49 & \\
\hline Marching $\left(3-4 \mathrm{~m} \mathrm{~h}^{-1}\right)$ & 11 & 6.03 & & 4.94 & \\
\hline Running (at the double in uniform) & 9 & 11.67 & & 9.59 & \\
\hline Stair-climbing (up and down 46 steps 7 inches high) & 3 & 10.3 & & 8.8 & \\
\hline Dressing & 9 & 4.0 & & 3.3 & \\
\hline Ironing (standing 51/2 lb iron) & 5 & 4.2 & & 3.51 & \\
\hline Polishing kit (sitting) & 7 & 2.4 & & 1.96 & \\
\hline Rifle cleaning & 6 & 2.7 & & 2.27 & \\
\hline Archery & 2 & 5.24 & & 4.36 & \\
\hline Batting (running twice between wickets every 6 balls) & 6 & 8.0 & & 6.35 & \\
\hline Bowling & 4 & 8.0 & & 6.35 & \\
\hline Squash & 6 & 10.18 & & 8.52 & \\
\hline Tennis & 7 & 7.13 & & 5.8 & \\
\hline Cycling $\left(12-13 \mathrm{~m} \mathrm{~h}^{-1}\right)$ & 8 & 7.72 & & 6.34 & \\
\hline Motor cycling (heavy army) & 3 & 2.82 & & 2.39 & \\
\hline Driving (army truck) & 3 & 3.38 & & 2.87 & \\
\hline Obstacle course & 6 & 6.16 & & 4.94 & \\
\hline Parade & 11 & 5.20 & & 4.26 & \\
\hline Weapon training & 12 & 2.21 & & 1.81 & \\
\hline
\end{tabular}

Abbreviations: BMR - basal metabolic rate; PAR - physical activity ratio.

Subjects: 12 British males (age 20 years, height $178 \mathrm{~cm}$, weight $68 \mathrm{~kg}$, all means).

Equipment: Douglas Bag/Max Planck Institute calorimeter with gas analysis.

PAR calculated from predicted BMR. 
Table 24 Edholm, 1973. Predominantly agricultural activities of Yemenite and Kurdisk Jews in Israel $\left.\right|^{27}$

\begin{tabular}{|c|c|c|c|c|}
\hline \multirow[b]{2}{*}{ Activities } & \multicolumn{2}{|c|}{$\begin{array}{c}\text { Energy } \\
\text { cost of activity } \\
\left(\mathrm{kcal} \mathrm{min}^{-1}\right)\end{array}$} & \multicolumn{2}{|c|}{ PAR } \\
\hline & Men & Women & Men & Women \\
\hline Tractor driving & 2.2 & & 1.9 & \\
\hline Truck driving & 1.9 & & 1.64 & \\
\hline Horse cart driving (standing) & 2.1 & & 1.81 & \\
\hline Potato picking & 6.58 & & 5.67 & \\
\hline Potato, filling sacks & 3.4 & & 2.93 & \\
\hline Potato, loading sacks on truck & 9.3 & & 8.01 & \\
\hline Potato grading & 3.15 & & 2.71 & \\
\hline Orange picking & 3.7 & & 3.19 & \\
\hline Weeding & 3.0 & & 2.58 & \\
\hline Picking carrots & 2.6 & & 2.24 & \\
\hline Seed casting & 4.5 & & 3.88 & \\
\hline Spray insecticide & 5.0 & & 4.31 & \\
\hline Manure spreading & 6.3 & & 5.43 & \\
\hline Prune vines & 4.05 & & 3.49 & \\
\hline Scythe grass & 5.9 & & 5.08 & \\
\hline Fork grass & 6.0 & & 5.17 & \\
\hline Sitting & 1.32 & & 1.14 & \\
\hline Walking & 4.0 & & 3.45 & \\
\hline Walking in mud & 8.0 & & 6.89 & \\
\hline Repair work with tractors & 4.5 & & 3.88 & \\
\hline Bicycling & 7.6 & & 6.55 & \\
\hline \multicolumn{5}{|l|}{ Housework } \\
\hline Wash and tidy & & 2.5 & & 2.75 \\
\hline Cook & & 2.0 & & 2.2 \\
\hline Scrub floor & & 3.2 & & 3.52 \\
\hline \multicolumn{5}{|l|}{ Animal work } \\
\hline Feed cows & & 3.4 & & 3.74 \\
\hline Feed chicken & & 3.1 & & 3.41 \\
\hline \multicolumn{5}{|l|}{ Field work } \\
\hline Weeding & & 3.32 & & 3.66 \\
\hline Top carrots & & 2.14 & & 2.36 \\
\hline Fork grass & & 4.5 & & 4.96 \\
\hline Sitting & & 1.36 & & 1.5 \\
\hline Walking and shopping & & 4.15 & & 4.57 \\
\hline
\end{tabular}

Abbreviations: BMR - basal metabolic rate; PAR - physical activity ratio. Subjects: numbers on whom energy costs were measured are not clear, ages between 20 and 30 years.

Equipment: mouth and noseclip with Max Planck respirometer or the Wright flowmeter. Gas analysis with Lloyd-Haldane apparatus.

Measurements: durations unclear.

PAR calculated using predicted BMR assuming a height of $1.72 \mathrm{~m}$ and weight of $65 \mathrm{~kg}$ for males and $1.62 \mathrm{~m}$ and $55 \mathrm{~kg}$ for females.
Table 25 Edmundson, 1989. Domestic and occupational activities in rural India ${ }^{28}$

\begin{tabular}{|c|c|c|c|c|c|}
\hline \multirow[b]{2}{*}{ Activities } & \multirow[b]{2}{*}{$n$} & \multicolumn{2}{|c|}{$\begin{array}{c}\text { Energy } \\
\text { cost of activity } \\
\left(\mathrm{kcal} \mathrm{kg}^{-1} \mathrm{~min}^{-1}\right)\end{array}$} & \multicolumn{2}{|c|}{ PAR } \\
\hline & & Men & Women & Men & Women \\
\hline Sitting & 24 & 0.026 & & 1.23 & \\
\hline Squatting & 6 & 0.027 & & 1.27 & \\
\hline Standing & 20 & 0.030 & & 1.42 & \\
\hline Strolling & 5 & 0.043 & & 2.03 & \\
\hline Walking & 16 & 0.054 & & 2.55 & \\
\hline Light work & 8 & 0.044 & & & \\
\hline Light carry & 4 & 0.092 & & 4.34 & \\
\hline Heavy carry & 7 & 0.119 & & 5.62 & \\
\hline Ploughing & 2 & 0.130 & & 6.14 & \\
\hline Medium work & 10 & 0.063 & & & \\
\hline Hoeing & 6 & 0.076 & & 3.59 & \\
\hline Heavy work & 6 & 0.110 & & & \\
\hline Cooking & 6 & & 0.040 & & 2.08 \\
\hline Housework & 4 & & 0.058 & & 3.01 \\
\hline
\end{tabular}

Abbreviations: BMR - basal metabolic rate; PAR - physical activity ratio. Subjects: Eight male (age 33.2 years, height $161.1 \mathrm{~cm}$, weight $48.3 \mathrm{~kg}$ per cent fat 17.0) and Eight females (age 28.1 years, height $148.8 \mathrm{~cm}$ weight $36.9 \mathrm{~kg}$, percent fat 21.2 , all means). Variable numbers for different activities.

Equipment: Kofranyi-Michaelis respirometer, with gas analysis using Micro-Scholander.

PAR calculated using predicted BMR.

Table 26 Fariduddin, 1975. Energy costs of Bangladeshi rickshaw-pullers and cart-pullers ${ }^{29}$

\begin{tabular}{|c|c|c|c|c|}
\hline \multirow[b]{2}{*}{ Activities } & \multicolumn{2}{|c|}{$\begin{array}{c}\text { Energy } \\
\text { cost of activity } \\
\left(\mathrm{kcal} \mathrm{min}^{-1}\right)\end{array}$} & \multicolumn{2}{|c|}{ PAR } \\
\hline & Men & Women & Men & Women \\
\hline \multicolumn{5}{|l|}{ Pedalling rickshaws } \\
\hline Without passengers & 6.66 & & 6.64 & \\
\hline With passengers & 7.84 & & 7.82 & \\
\hline \multicolumn{5}{|l|}{ Cart pulling } \\
\hline Without load & 5.5 & & 5.68 & \\
\hline With load & 6.08 & & 6.28 & \\
\hline
\end{tabular}

Abbreviations: BMR - basal metabolic rate; PAR - physical activity ratio. Subjects: 10 rickshaw-pullers (age 26 years, height $5.26 \mathrm{ft}$, weight $50 \mathrm{~kg}$ ) and 11 cart-pullers (age 25 years, height $5.5 \mathrm{ft}$, weight $46.8 \mathrm{~kg}$, all means). Equipment: Douglas bag. Dry gas meter calibration against Tissot's. Lloyd's modification of the Haldane apparatus for $\mathrm{O}_{2}$ and $\mathrm{CO}_{2}$. PAR calculated using predicted BMR.

Table 27 Fariduddin, 1976. Energy cost of some common activities in Bangladesh ${ }^{30}$

\begin{tabular}{|c|c|c|c|c|}
\hline \multirow[b]{2}{*}{ Activities } & \multicolumn{2}{|c|}{$\begin{array}{l}\text { Energy cost of activity } \\
\left.\qquad(\mathrm{kcal} \mathrm{min})^{-1}\right)\end{array}$} & \multicolumn{2}{|c|}{ PAR } \\
\hline & Men & Women & Men & Women \\
\hline 10 cultivators: ploughing & 5.45 & & 5.17 & \\
\hline 12 carpenters: wood planning & 4.24 & & 4.41 & \\
\hline 6 labourers: Earth-cutting in a brick field & 5.19 & & 5.52 & \\
\hline 6 labourers: brick-breaking & 3.4 & & 3.51 & \\
\hline
\end{tabular}

Abbreviations: BMR - basal metabolic rate; PAR - physical activity ratio.

Subjects: ? male divided into four groups; 10 cultivators (age 29.7 years, height $169.8 \mathrm{~cm}$, weight $54.9 \mathrm{~kg}$ ), 12 carpenters (age 28.6 years, height $159.3 \mathrm{~cm}$, weight $46.1 \mathrm{~kg}$ ), six labourers (age 25.5 years, height $163.7 \mathrm{~cm}$, weight $44.3 \mathrm{~kg}$ ) and six labourers for brick-breaking (age 25 years, height $164 \mathrm{~cm}$, weight $46.8 \mathrm{~kg}$, all means).

Equipment: Douglas bag. Calibrated dry gas meter and Lloyd's modification of Haldane's gas analysis. Expired air collected at the height of activity.

PAR calculated using predicted BMR. 
Table 28 Garby, 1987. Energy expenditure during sleep ${ }^{31}$

\begin{tabular}{|c|c|c|c|c|}
\hline \multirow[b]{2}{*}{ Activities } & \multicolumn{2}{|c|}{$\begin{array}{l}\text { Energy cost } \\
\text { of activity } \\
\left(\mathrm{kcal} \mathrm{min}^{-1}\right)\end{array}$} & \multicolumn{2}{|c|}{ PAR } \\
\hline & Men & Women & Men & Women \\
\hline Sleep & & & 0.97 & 0.94 \\
\hline
\end{tabular}

Abbreviations: BMR - basal metabolic rate; PAR - physical activity ratio. Subjects: 59 subjects ( 38 male, 21 female). Males (age 24 years, weight $66.6 \mathrm{~kg}$, per cent fat 12.4), Females (age 26 years, weight $64.2 \mathrm{~kg}$, per cent fat 27.7 , all means).

Equipment: previously validated whole body direct calorimeter.

BMR measured as (Watt $\mathrm{h}^{-1}$ ) males 85.6/1.3 SE, females 71.2/1.09 SE

Measurement: sleeping metabolic rate measured between 11:30 and 6:30

$(7 \mathrm{~h})$, subjects went to sleep at 11:00.

PAR as computed by the authors.

Table 29 Haisman, 1972. Energy cost of pushing a variety of loaded $(50 \mathrm{~kg})$ hand carts during a 30 minute walk ${ }^{32}$

\begin{tabular}{llllll}
\hline & \multicolumn{2}{c}{$\begin{array}{c}\text { Energy cost of } \\
\text { activity (Watt) }\end{array}$} & & \multicolumn{2}{c}{ PAR } \\
\cline { 2 - 2 } Activities & Men & Women & & Men & Women \\
\hline Mail cart & 478 & & 5.22 & \\
Large garden cart & 493 & & 5.42 & \\
Small garden cart & 524 & & 5.76 & \\
Golf cart & 551 & & 6.06 & \\
\hline
\end{tabular}

Abbreviations: BMR - basal metabolic rate; PAR - physical activity ratio. Subjects: 7 healthy soldiers (age 20.7 years, height $178.6 \mathrm{~cm}$, weight $78.5 \mathrm{~kg}$, all means).

Equipment: Max Planck respirometer. Gas analysis using Beckman E2 $\mathrm{O}_{2}$ analyser and $\mathrm{LB} 1 \mathrm{CO}_{2}$ analyser.

Measurement: data here is restricted to values obtained on an outdoor circuit. Data were also collected on a treadmill.

PAR calculated using predicted BMR.

Table 30 Igbanugo, 1978. Energy cost of aerobic dancing ${ }^{33}$

\begin{tabular}{|c|c|c|c|c|}
\hline \multirow[b]{2}{*}{ Activities } & \multicolumn{2}{|c|}{$\begin{array}{l}\text { Energy cost } \\
\text { of activity } \\
\left(\mathrm{kcal} \mathrm{min}^{-1}\right)\end{array}$} & \multicolumn{2}{|c|}{ PAR } \\
\hline & Men & Women & Men & Women \\
\hline \multirow[t]{2}{*}{ Low intensity aerobics } & 4.07 & 4.04 & 3.68 & 4.45 \\
\hline & 4.26 & 3.87 & 3.33 & 4.03 \\
\hline \multirow[t]{2}{*}{ Medium intensity aerobics } & 6.58 & 5.83 & 5.94 & 6.42 \\
\hline & 7.15 & 6.74 & 5.58 & 7.03 \\
\hline \multirow[t]{2}{*}{ High intensity aerobics } & 9.2 & 7.73 & 8.31 & 8.54 \\
\hline & 9.67 & 7.74 & 7.55 & 8.07 \\
\hline
\end{tabular}

Abbreviations: BMR - basal metabolic rate; PAR - physical activity ratio. Subjects: Two men and two women, non-dancers. Individual anthropometry provided.

Equipment: Max Planck respirometer with gas analysers: Beckman for $\mathrm{O}_{2}$ and Harvard apparatus for $\mathrm{CO}_{2}$.

Measurement: three levels of aerobic dancing; seven routines at each intensity. Each routine 2-3 minutes alternated with recovery periods of 15-90 seconds. Metabolic values include both dance and recovery.

PAR calculations based on predicted BMR.
Table 31 Jette, 1979. Energy costs of rope skipping ${ }^{34}$

\begin{tabular}{|c|c|c|c|}
\hline \multirow[b]{2}{*}{ Activities } & \multicolumn{2}{|c|}{$\begin{array}{l}\text { Energy cost } \\
\text { of activity } \\
\left(\mathrm{kcal} \mathrm{h} \mathrm{kg}^{-1}\right)\end{array}$} & PAR \\
\hline & Men & Women & Men \\
\hline 66 TPM, 2 ft skip, rhythm bounce & 9.13 & & 8.68 \\
\hline 102 TPM, 2 ft skip, plain bounce & 12.39 & & 11.78 \\
\hline
\end{tabular}

Abbreviations: BMR - basal metabolic rate; PAR - physical activity ratio. TPM - turns per minute.

Subjects: Five Canadian males, well conditioned but with minimal experience with rope skipping (age 26.6 years, height $172.5 \mathrm{~cm}$, weight $68.1 \mathrm{~kg}$, all means).

Equipment: Tissot gasometer, Godart-Statham paramagnetic $\mathrm{O}_{2}$ analyser and $\mathrm{a} \mathrm{G}-\mathrm{S} \mathrm{CO}_{2}$ analyser.

Measurement: rope skipping at different levels of intensity: 6-132 turns per minute, each skipping bout: 5 minutes. Expired air collected for 30 second in 3rd and 5th minute.

PAR calculated based on the predicted BMR.

Table 32 Lawrence, 1985. Energy cost of a variety of common daily activities ${ }^{35}$

\begin{tabular}{|c|c|c|c|c|c|}
\hline \multirow[b]{2}{*}{ Activities } & \multirow[b]{2}{*}{$n$} & \multicolumn{2}{|c|}{$\begin{array}{l}\text { Energy cost } \\
\text { of activity } \\
\left(\mathrm{kcal} \mathrm{min}^{-1}\right)\end{array}$} & \multicolumn{2}{|c|}{ PAR } \\
\hline & & Men & Women & Men & Women \\
\hline Shelling ground nuts & 37 & 1.38 & & 1.59 & \\
\hline Plaiting hair & 21 & 1.52 & & 1.75 & \\
\hline Sorting groundnuts & 13 & 1.62 & & 1.87 & \\
\hline Weeding rice & 45 & 1.98 & & 2.28 & \\
\hline Harvesting rice & 19 & 2.04 & & 2.35 & \\
\hline Weeding groundnuts & 25 & 2.81 & & 3.24 & \\
\hline Drawing water & 89 & 2.91 & & 3.36 & \\
\hline Beating groundnuts & 15 & 3.06 & & 3.53 & \\
\hline Washing clothes & 29 & 3.26 & & 3.76 & \\
\hline Bending digging & 21 & 4.05 & & 4.67 & \\
\hline Harvesting groundnuts & 17 & 4.07 & & 4.69 & \\
\hline Standing digging & 14 & 4.72 & & 5.44 & \\
\hline Pounding grain & 54 & 5.02 & & 5.79 & \\
\hline
\end{tabular}

Abbreviations: BMR - basal metabolic rate; PAR - physical activity ratio. Subjects: women, variable numbers for different activities (age: most from 18 to 35 years). Women consisted of non-pregnant, non-lactating women (average weight $49.8 \mathrm{~kg}$ ), Pregnant women in the first trimester (average weight $50.4 \mathrm{~kg}$ ) and lactating mothers (average weight $53.3 \mathrm{~kg}$ ).

Measurement: made using Douglas bags. Gas collections for 5 minutes after a 3 minute equilibrium period. Gas analysis with calibrated analysers. Volume with a wet gas meter.

PAR calculated using predicted BMR.

Table 33 Lemon, 1977. Energy cost of fire fighting ${ }^{36}$

\begin{tabular}{|c|c|c|c|c|}
\hline \multirow[b]{2}{*}{ Activities } & \multicolumn{2}{|c|}{$\begin{array}{l}\text { Energy cost } \\
\text { of activity } \\
\left(\mathrm{kcal} \mathrm{min}^{-1}\right)\end{array}$} & \multicolumn{2}{|c|}{ PAR } \\
\hline & Men & Women & Men & Women \\
\hline Ariel LD climb & $11-12.2$ & & 8.89 & \\
\hline Rescue victim & 12.7 & & 9.67 & \\
\hline Dragging hose & 12.7 & & 9.79 & \\
\hline Ladder raise & 11.5 & & 8.76 & \\
\hline
\end{tabular}

Abbreviations: BMR - basal metabolic rate; PAR - physical activity ratio. Subjects: 20 male professional firefighters, anthropometric profiles for each activity provided by the authors.

Measurement: expired gas volumes collected and aliquots measured using a Gallenkamp-lloyd Gas analyser. Each activity was on ? five subjects repeated on two separate occasions.

PAR based on predicted BMR. 
Table 34 Li Jing, 1991. Energy cost of 'college activities'37

\begin{tabular}{|c|c|c|c|c|}
\hline \multirow[b]{2}{*}{ Activities } & \multicolumn{2}{|c|}{$\begin{array}{c}\text { Energy cost } \\
\text { of activity } \\
\left(\mathrm{kcal} \mathrm{m}^{2} \mathrm{~min}^{-1}\right)\end{array}$} & \multicolumn{2}{|c|}{ PAR } \\
\hline & Men & Women & Men & Women \\
\hline Reading in bed & 0.813 & 0.801 & 1.22 & 1.22 \\
\hline Sitting and thinking & 0.839 & 0.818 & 1.25 & 1.25 \\
\hline Standing silently & 0.886 & 0.846 & 1.32 & 1.29 \\
\hline Self-study & 0.906 & 0.904 & 1.35 & 1.38 \\
\hline Watch TV & 1.100 & 1.129 & 1.64 & 1.72 \\
\hline Listen to music & 0.970 & 0.939 & 1.45 & 1.43 \\
\hline Painting & 0.838 & 0.835 & 1.25 & 1.27 \\
\hline Play musical instruments & 1.346 & & 2.01 & \\
\hline Play chess & 0.930 & 1.006 & 1.39 & 1.53 \\
\hline Play cards & 1.219 & 1.315 & 1.82 & 2.0 \\
\hline Watching a laboratory experiment & 0.890 & 0.850 & 1.33 & 1.3 \\
\hline Carrying on animal experiment & 1.262 & 1.382 & 1.88 & 2.11 \\
\hline Listening to lecture & 1.146 & 1.068 & 1.71 & 1.63 \\
\hline \multicolumn{5}{|l|}{ Leisure time } \\
\hline Walking & 2.608 & 2.561 & 3.9 & 3.9 \\
\hline Riding on bicycle & 2.542 & 2.372 & 3.62 & 3.62 \\
\hline Exercising on horizontal bar & 3.490 & 3.426 & 5.22 & 5.22 \\
\hline Long distance running & 3.202 & 2.666 & 4.79 & 4.06 \\
\hline Dancing fast & 4.221 & 4.293 & 6.31 & 6.54 \\
\hline Dancing moderate & 3.640 & 3.463 & 5.44 & 5.28 \\
\hline Dancing slowly & 2.199 & 2.266 & 3.29 & 3.45 \\
\hline Tennis & 3.905 & 3.884 & 5.84 & 5.92 \\
\hline Volleyball & 4.054 & 3.977 & 6.06 & 6.06 \\
\hline Basket ball & 4.652 & 5.078 & 6.95 & 7.74 \\
\hline Football & 5.671 & & 8.48 & \\
\hline Sprint & 5.490 & 5.433 & 8.21 & 8.28 \\
\hline Push-up & 3.490 & 3.426 & 5.22 & 5.22 \\
\hline Sewing & & 1.188 & & 1.81 \\
\hline Skill gym & & 3.210 & & 4.89 \\
\hline Middle distance running & & 4.010 & & 6.11 \\
\hline
\end{tabular}

Abbreviations: BMR - basal metabolic rate; PAR - physical activity ratio. Subjects: numbers for each activity not given - 606 subjects randomly selected from Shanghai Medical University (319 males, 287 females, age range 18-24 years, no anthropometry available).

Measurements: Douglas bag with volume, and gas measurements - S-3A $\mathrm{O}_{2}, \mathrm{H} \& \mathrm{~B} \mathrm{CO}$.

PAR is what has been provided in the text.
Table 35 Littell, 1969. Energy costs of flying ${ }^{38}$

\begin{tabular}{|c|c|c|c|c|}
\hline \multirow[b]{2}{*}{ Activities } & \multicolumn{2}{|c|}{$\begin{array}{l}\text { Energy cost } \\
\text { of activity } \\
\left(\text { kcal min }^{-1}\right)\end{array}$} & \multicolumn{2}{|c|}{ PAR } \\
\hline & Men & Women & Men & Women \\
\hline \multicolumn{5}{|l|}{ Light helicopter } \\
\hline BMR $n=8$ & 1.18 & & & \\
\hline Sitting (5 min) & 1.74 & & 1.47 & \\
\hline Hover/Taxi (4 min) & 2.17 & & 1.84 & \\
\hline Ascend (2 min) & 2.06 & & 1.75 & \\
\hline Level 1 (5 min) & 1.60 & & 1.36 & \\
\hline Aerobatics (7 min) & 1.61 & & 1.36 & \\
\hline Level 2 (5 min) & 1.50 & & 1.27 & \\
\hline Descend (2 min) & 1.75 & & 1.48 & \\
\hline \multicolumn{5}{|l|}{ Utility helicopter } \\
\hline Basal (BMR) $n=8$ & 1.19 & & & \\
\hline Sitting (5 min) & 1.60 & & 1.34 & \\
\hline Hover/taxi (4 min) & 1.76 & & 1.48 & \\
\hline Ascend (2 min) & 1.69 & & 1.42 & \\
\hline Level 1 (5 min) & 1.58 & & 1.32 & \\
\hline Aerobatics (7 min) & 1.54 & & 1.29 & \\
\hline Level 2 (5 min) & 1.42 & & 1.19 & \\
\hline Descend (2 min) & 1.52 & & 1.28 & \\
\hline \multicolumn{5}{|l|}{ Medium helicopter } \\
\hline Basal (BMR) $n=7$ & 1.21 & & & \\
\hline Sitting (5 min) & 1.60 & & 1.32 & \\
\hline Hover/taxi (4 min) & 2.03 & & 1.68 & \\
\hline Ascend (2 min) & 2.03 & & 1.68 & \\
\hline Level 1 (5 min) & 1.71 & & 1.41 & \\
\hline Aerobatics (7 min) & 1.73 & & 1.43 & \\
\hline Level 2 (5 min) & 1.67 & & 1.38 & \\
\hline Descend (2 min) & 1.85 & & 1.53 & \\
\hline \multicolumn{5}{|c|}{ Fixed wing utility aircraft } \\
\hline $\operatorname{BMR} n=4$ & 1.04 & & & \\
\hline Sitting (5 min) & 1.72 & & 1.65 & \\
\hline Hover/taxi (4 min) & 2.69 & & 2.58 & \\
\hline Ascend (2 min) & 2.78 & & 2.67 & \\
\hline Level 1 (5 min) & 1.87 & & 1.8 & \\
\hline Aerobatics (7 min) & 1.76 & & 1.69 & \\
\hline Level 2 (5 min) & 1.70 & & 1.63 & \\
\hline Descend (2 min) & 2.43 & & 2.34 & \\
\hline
\end{tabular}

Abbreviations: BMR - basal metabolic rate; PAR - physical activity ratio. Subjects: 16 experienced aviators (age 39.5 years, height $176.1 \mathrm{~cm}$, weight $78.7 \mathrm{~kg}$, all mean).

Equipment: expired air collected through a facemask, connected to a Mueller Franz gas meter for volume and a paramagnetic $\mathrm{O}_{2}$ analyser (Beckman model E-2).

PAR calculated using measured BMR.

Table 36 Louhevaara, 1988. Sorting of postal parcels, in a simulated work place ${ }^{39}$

\begin{tabular}{|c|c|c|c|c|}
\hline \multirow[b]{2}{*}{ Activities } & \multicolumn{2}{|c|}{$\begin{array}{c}\text { Energy cost of } \\
\text { activity }\left(\mathrm{VO}_{2} \text { litre } \min ^{-1}\right)\end{array}$} & \multicolumn{2}{|c|}{ PAR } \\
\hline & Men & Women & Men & Women \\
\hline $\begin{array}{l}\text { Slow sorting ( } 4 \mathrm{~min} \text { at each level of activity) } 3 \text { parcels per minute, } \\
\text { walking speed } 0.3 \mathrm{~m} \mathrm{~s}^{-1} \text {. (Wt of parcels } 15.3 \mathrm{~kg} \mathrm{~min}^{-1} \text { ) }\end{array}$ & 1.03 & & 4.09 & \\
\hline Habitual sorting ( 8.6 parcels per minute, $0.6 \mathrm{~m} \mathrm{~s}^{-1} 43.9 \mathrm{~kg} \mathrm{~min}^{-1}$ ) & 1.36 & & 5.4 & \\
\hline $\begin{array}{l}\text { Accelerated sorting ( } 10.8 \text { parcels per minute, } \\
\left.0.7 \mathrm{~m} \mathrm{~s}^{-1} 55.1 \mathrm{~kg} \mathrm{~min}^{-1}\right)\end{array}$ & 1.80 & & 7.14 & \\
\hline Maximal sorting (16.9 parcels per minute, $1.0 \mathrm{~m} \mathrm{~s}^{-1}, 86.2 \mathrm{~kg} \mathrm{~min}^{-1}$ ) & 2.18 & & 8.65 & \\
\hline
\end{tabular}

Abbreviations: BMR - basal metabolic rate; PAR - physical activity ratio.

Subjects: 21 healthy male sorters of postal parcels (age 33.3 years, height $178.4 \mathrm{~cm}$, weight $78.3 \mathrm{~kg}$, per cent fat 18.4).

Equipment: microprocessor controlled respiratory gas analyser (Morgan 500d), using a paramagnetic $\mathrm{O}_{2}$, and infrared $\mathrm{CO}_{2}$ analyser.

Measurement: 100 parcels of standard weight had to be sorted and placed in two trolleys based on postal code.

PAR based on predicted BMR. 
Table 37 Malhotra, 1976. Energy cost of a variety of activities in Indian submariners ${ }^{40}$

\begin{tabular}{|c|c|c|c|}
\hline \multirow[b]{2}{*}{ Activities } & \multicolumn{2}{|c|}{$\begin{array}{c}\text { Energy } \\
\text { cost of activity } \\
\left(\mathrm{kJ} \mathrm{min}^{-1}\right)\end{array}$} & PAR \\
\hline & Men & Women & Men Women \\
\hline Sleep & 4.35 & & 0.94 \\
\hline Rest and relaxation & 4.85 & & 1.04 \\
\hline Reading and writing & 5.02 & & 1.08 \\
\hline Standing & 5.31 & & 1.15 \\
\hline Eating and drinking & 6.27 & & 1.35 \\
\hline $\begin{array}{l}\text { Equipment operation, action } \\
\text { station, on duty, watch keeping }\end{array}$ & 8.28 & & 1.79 \\
\hline Toilet and dressing & 8.36 & & 1.81 \\
\hline Equipment cleaning & 12.12 & & 2.62 \\
\hline Ascending and descending ladders & 16.01 & & 3.46 \\
\hline Walking between compartments & 16.55 & & 3.58 \\
\hline Loading and unloading & 16.55 & & 4.35 \\
\hline Clean ship: done at surface & 24.66 & & 5.33 \\
\hline
\end{tabular}

Abbreviations: BMR - basal metabolic rate; PAR - physical activity ratio. Subjects: numbers uncertain (age 26 years, weight $59.8 \mathrm{~kg}$, height $168.8 \mathrm{~cm})$.

Equipment: Kofranyi-Michaelis respirometers with Scholander micro-gas analysers.

Measurements: done at 'steady state'.

PAR based on predicted BMR.

Table 38 Marchetti, 1980. Energy cost of sailing ${ }^{41}$

\begin{tabular}{lccccc}
\hline & \multicolumn{2}{c}{$\begin{array}{c}\text { Energy cost } \\
\text { of activity } \\
\left(\mathrm{VO}_{2} \text { in } \mathrm{ml} \mathrm{min}^{-1}\right)\end{array}$} & & \multicolumn{2}{c}{ PAR } \\
\cline { 2 - 3 } \cline { 5 - 6 } Activities & Men & Women & & Men & Women \\
\hline $\begin{array}{c}\text { Energy cost } \\
\text { of sailing }\end{array}$ & 340 & 283 & & 1.42 & 1.54 \\
& 340 & & 1.42 & \\
\hline
\end{tabular}

Abbreviations: BMR - basal metabolic rate; PAR - physical activity ratio. Subjects: Three Caucasian subjects: two male and one female (individual anthropometric details given in the paper.

Equipment: Douglas bags and Haldane-Margaria gas analyser.

Measurement: use of trapeze which allows subject to extend body outboard. Only data on the 'lake' are used here. Simulated lab conditions not used. $\mathrm{VO}_{2}$ collected for 5 minutes at steady state, 5 minutes after start of routine.

PAR calculated based on predicted BMRs.
Table 39 Montgomery, 1977. Energy costs of activities of Amazon Indian hunter-gatherer-horticulturalists ${ }^{42}$

\begin{tabular}{|c|c|c|c|c|c|}
\hline \multirow[b]{2}{*}{ Activities } & \multirow[b]{2}{*}{$n$} & \multicolumn{2}{|c|}{$\begin{array}{l}\text { Energy cost } \\
\text { of activity } \\
\left(\mathrm{kcal} \mathrm{min}^{-1}\right)\end{array}$} & \multicolumn{2}{|c|}{ PAR } \\
\hline & & Men & Women & Men & Women \\
\hline Walking open foot paths & 6 & 5.3 & & 5.1 & \\
\hline Walking level forest paths & 6 & 6.0 & & 5.78 & \\
\hline Walking up forest paths & 9 & 8.9 & & 8.57 & \\
\hline Walking down forest paths & 3 & 4.1 & & 3.95 & \\
\hline Clearing undergrowth & 5 & 7.3 & & 7.03 & \\
\hline Felling large trees & 8 & 7.6 & & 7.32 & \\
\hline Planting maize & 2 & 4.3 & & 4.14 & \\
\hline Planting manioc & 7 & 5.2 & & 5.01 & \\
\hline Weeding slope & 4 & 6.1 & & 5.87 & \\
\hline Cutting grass & 1 & 7.0 & & 6.74 & \\
\hline Harvesting maize & 5 & 5.3 & & 5.1 & \\
\hline Harvesting manioc & 4 & 4.4 & & 4.24 & \\
\hline Removing palmheart & 1 & 6.2 & & 5.97 & \\
\hline Chopping firewood logs & 2 & 6.7 & & 6.45 & \\
\hline Net bag manufacture & 9 & 2.6 & & 2.5 & \\
\hline Cane box manufacture & 2 & 2.2 & & 2.12 & \\
\hline Bow and arrow manufacture & 15 & 2.7 & & 2.6 & \\
\hline Planting root crops & 2 & & 2.9 & & 3.69 \\
\hline Harvesting root crops & 7 & & 2.7 & & 3.43 \\
\hline Catching fish with hands & 4 & & 3.1 & & 3.94 \\
\hline Weeding yard & 2 & & 2.4 & & 3.05 \\
\hline Sweeping yard & 1 & & 2.8 & & 3.56 \\
\hline Deseeding cotton & 2 & & 1.4 & & 1.78 \\
\hline Beating cotton & 4 & & 1.9 & & 2.42 \\
\hline Spinning cotton & 6 & & 1.1 & & 1.4 \\
\hline Setting loom & 2 & & 2.0 & & 2.54 \\
\hline Weaving & 8 & & 1.8 & & 2.29 \\
\hline Grinding maize & 6 & & 2.8 & & 3.56 \\
\hline Peeling manioc & 3 & & 2.1 & & 2.67 \\
\hline Splitting manioc & 2 & & 2.0 & & 2.54 \\
\hline Straining manioc & 6 & & 1.9 & & 2.42 \\
\hline Washing laundry & 3 & & 2.6 & & 3.31 \\
\hline
\end{tabular}

Abbreviations: BMR - basal metabolic rate; PAR - physical activity ratio. Subjects: Eight men (age 25 years, weight $51.8 \mathrm{~kg}$ ) and eight women (age 25 years, weight $44.5 \mathrm{~kg}$, all means). Variable numbers for different studies.

Equipment: Max Planck respirometer with a fuel cell powered Teledyne oxygen analyser.

PAR based on predicted BMR. 
Table 40 Nag, 1980. Various agricultural tasks in India ${ }^{43}$

\begin{tabular}{|c|c|c|c|c|}
\hline \multirow[b]{2}{*}{ Activities } & \multicolumn{2}{|c|}{$\begin{array}{l}\text { Energy cost of } \\
\text { activity }\left(\mathrm{kJ} \mathrm{min}^{-1}\right)\end{array}$} & \multicolumn{2}{|c|}{ PAR } \\
\hline & Men & Women & Men & Women \\
\hline \multicolumn{5}{|l|}{ Seeding operations } \\
\hline Sitting, resting & $4.25 / 0.86$ & & 1.01 & \\
\hline Free walking on plane surface & $11.21 / 1.06$ & & 2.68 & \\
\hline Free walking on puddle field & $13.73 / 0.53$ & & 3.28 & \\
\hline Transplanting, bending on puddle field & $13.01 / 0.93$ & & 3.1 & \\
\hline Germinating seeder & $34.42 / 2.34$ & & 8.21 & \\
\hline $\begin{array}{l}\text { Germinating seeder (IRRI type) The IRRI seeder consists } \\
\text { of eight concentric aluminium pipes running upwards } \\
\text { to a hopper, i.e. the germinating seed reservoir. } \\
\text { The distance between the pipes at the ground level is } \\
\text { about } 20 \mathrm{~cm} \text {. As the structure is pulled manually on a } \\
\text { chain wheel a flap of the reservoir is opened to the } \\
\text { pipes through which seeds come out and fall } \\
\text { on the ground }\end{array}$ & $40.2 / 3.64$ & & 9.59 & \\
\hline \multicolumn{5}{|l|}{ Threshing operation } \\
\hline Manual threshing by beating & 19.26/1.18 & & 4.6 & \\
\hline Pedal threshing & $27.56 / 3.25$ & & 6.58 & \\
\hline Pedal threshing, helper & $13.53 / 1.99$ & & 3.23 & \\
\hline
\end{tabular}

Abbreviations: BMR - basal metabolic rate; IRRI - International Rice Research Institute; PAR - physical activity ratio.

Five male agricultural workers (age 23.4, height $164.6 \mathrm{~cm}$, weight $49.9 \mathrm{~kg}$, all means).

Equipment: $\mathrm{KM}$ respirometer linked to a Beckman paramagnetic $\mathrm{O}_{2}$ analyser.

At 'steady state' measurements were made.

PAR calculated using predicted BMR.

Table 41 Nag, 1981. Variety of household and agricultural tasks ${ }^{44}$

\begin{tabular}{|c|c|c|c|c|}
\hline \multirow[b]{2}{*}{ Activities } & \multicolumn{2}{|c|}{$\begin{array}{l}\text { Energy cost of } \\
\text { activity }\left(\mathrm{kJ} \mathrm{min}{ }^{-1}\right)\end{array}$} & \multicolumn{2}{|c|}{ PAR } \\
\hline & Men & Women & Men & Women \\
\hline Squatting on floor washing utensils & & 5.42 & & 1.57 \\
\hline Kneeling down and working (e.g. sweeping floor) & & 7.89 & & 2.29 \\
\hline Making bed at 20 inches height & & 11.7 & & 3.41 \\
\hline Spreading grains/veg on the floor & & 15.05 & & 4.36 \\
\hline Winnowing (sitting) & & 8.57 & & 2.49 \\
\hline Walking & & 11.48 & & 3.33 \\
\hline Weeding with sickle (sitting) & & 11.54 & & 3.35 \\
\hline Uprooting (sitting) & & 11.96 & & 3.47 \\
\hline Harvesting paddy field (sitting) & & 12.17 & & 3.53 \\
\hline Transplanting paddy seedlings & & 12.33 & & 3.575 \\
\hline Harvesting (bending) & & 12.78 & & 3.71 \\
\hline Weeding using sickle (bending) & & 14.45 & & 4.189 \\
\hline Uprooting (bending) & & 15.5 & & 4.49 \\
\hline Digging dry soil using spade & & 19.5 & & 5.65 \\
\hline Pounding (single woman) & & 21.74 & & 6.3 \\
\hline Pounding (two women) & & 19.19 & & 5.56 \\
\hline
\end{tabular}

Abbreviations: BMR - basal metabolic rate; PAR - physical activity ratio.

Subjects: Indian females $n=8$, (age 30.4 years, weight $41.2 \mathrm{~kg}$, height $149.4 \mathrm{~cm}$, all means)

Equipment: open-circuit respirometer linked to paramagnetic $\mathrm{O}_{2}$ analyser.

PAL based on predicted BMR. 
Table 42 Norgan, 1974. Large range of activities measured on 204 New Guinean adults living in two villages ${ }^{45}$

\begin{tabular}{|c|c|c|c|c|c|}
\hline \multirow[b]{2}{*}{ Activities } & \multirow[b]{2}{*}{$n$} & \multicolumn{2}{|c|}{$\begin{array}{c}\text { Energy cost } \\
\text { of activity } \\
\left(\text { kcal min }^{-1}\right)\end{array}$} & \multicolumn{2}{|c|}{ PAR } \\
\hline & & Men & Women & Men & Women \\
\hline \multicolumn{6}{|l|}{ KAUL men } \\
\hline Lying & 42 & 1.14 & & 1.05 & \\
\hline Sitting & 41 & 1.23 & & 1.13 & \\
\hline Standing & 40 & 1.32 & & 1.21 & \\
\hline \multicolumn{6}{|l|}{ All sitting } \\
\hline Sitting weaving bamboo mat & 1 & 1.4 & & 1.29 & \\
\hline Sitting: tie morotta & 1 & 1.5 & & 1.38 & \\
\hline Separating copra and shell & 1 & 1.8 & & 1.66 & \\
\hline Sew morotta & 2 & 1.9 & & 1.75 & \\
\hline Carve plate, drum or comb & 3 & 2.0 & & 1.84 & \\
\hline Fish from canoe & 2 & 2.1 & & 1.94 & \\
\hline Weave bamboo wall & 2 & 2.8 & & 2.58 & \\
\hline Cut copra & 1 & 3.1 & & 2.86 & \\
\hline Paddle canoe & 2 & 3.3 & & 3.04 & \\
\hline \multicolumn{6}{|l|}{ All standing } \\
\hline Clean gun & 1 & 1.6 & & 1.47 & \\
\hline Mend lamp & 1 & 1.9 & & 1.75 & \\
\hline Fish with line & 1 & 2.0 & & 1.84 & \\
\hline Tie fence & 1 & 2.0 & & 1.84 & \\
\hline Plant tobacco & 1 & 2.3 & & 2.12 & \\
\hline Chop firewood & 1 & 2.5 & & 2.3 & \\
\hline Fish with spear & 1 & 2.5 & & 2.3 & \\
\hline Work in store & 1 & 2.6 & & 2.4 & \\
\hline Prune cocoa & 1 & 2.6 & & 2.4 & \\
\hline Cut tobacco & 1 & 2.7 & & 2.49 & \\
\hline Clear light bush & 4 & 2.8 & & 2.58 & \\
\hline Disbud tobacco & 1 & 2.9 & & 2.67 & \\
\hline Weed with shovel or hoe & 2 & 3.1 & & 2.86 & \\
\hline At 'sing-sing' & 1 & 3.1 & & 2.86 & \\
\hline Make fence & 1 & 3.5 & & 3.23 & \\
\hline Collect Daka (piper) & 1 & 3.6 & & 3.32 & \\
\hline Cycling & 1 & 4.5 & & 4.15 & \\
\hline Cut saplings & 3 & 4.0 & & 3.69 & \\
\hline Walking & 37 & 4.2 & & 3.87 & \\
\hline Walking slowly & 17 & 3.3 & & 3.04 & \\
\hline Walking around & 15 & 2.4 & & 2.21 & \\
\hline Weeding & 4 & 2.7 & & 2.49 & \\
\hline Clean garden & 2 & 3.1 & & 2.86 & \\
\hline Cut grass & 17 & 5.7 & & 5.25 & \\
\hline Collecting coconuts & 2 & 4.5 & & 4.15 & \\
\hline Husking coconuts & 4 & 6.1 & & 5.62 & \\
\hline Bag coconuts & 3 & 3.9 & & 3.59 & \\
\hline Bag and split coconut & 6 & 4.2 & & 3.87 & \\
\hline Hunting flying fox & 2 & 3.2 & & 2.95 & \\
\hline Hunting pigs & 2 & 3.5 & & 3.23 & \\
\hline \multicolumn{6}{|l|}{ House building } \\
\hline Cut bamboo & 1 & 3.1 & & 2.86 & \\
\hline Cut limbom trunks & 2 & 4.0 & & 3.69 & \\
\hline Collect bom bom & 1 & 4.0 & & 3.69 & \\
\hline Dig post-holes & 1 & 6.0 & & 5.53 & \\
\hline Lay floor & 1 & 4.0 & & 3.69 & \\
\hline Nailing & 1 & 3.2 & & 2.95 & \\
\hline \multicolumn{6}{|l|}{ KAUL women } \\
\hline Lying & 41 & & 1.03 & & 1.19 \\
\hline Sitting & 41 & & 1.08 & & 1.25 \\
\hline Standing & 41 & & 1.19 & & 1.38 \\
\hline \multicolumn{6}{|l|}{ Sitting or squatting activities } \\
\hline Sewing & 2 & & 1.2 & & 1.39 \\
\hline Prepare tobacco & 3 & & 1.3 & & 1.50 \\
\hline Remove beans & 2 & & 1.3 & & 1.50 \\
\hline Split cocoa & 1 & & 1.7 & & 1.97 \\
\hline Break galips & 4 & & 1.6 & & 1.85 \\
\hline Squeeze coconut & 2 & & 2.1 & & 2.43 \\
\hline Weaving bilum & 6 & & 1.2 & & 1.39 \\
\hline Preparing rope & 6 & & 1.3 & & 1.5 \\
\hline
\end{tabular}

Table 42 Continued

\begin{tabular}{|c|c|c|c|c|c|}
\hline \multirow[b]{2}{*}{ Activities } & \multirow[b]{2}{*}{$n$} & \multicolumn{2}{|c|}{$\begin{array}{l}\text { Energy cost } \\
\text { of activity } \\
\left(\text { kcal min }^{-1}\right)\end{array}$} & \multicolumn{2}{|c|}{ PAR } \\
\hline & & Men & Women & Men & Women \\
\hline Peeling taro & 33 & & 1.5 & & 1.73 \\
\hline \multicolumn{6}{|l|}{ Standing activities } \\
\hline Collect tulip leaves & 1 & & 1.6 & & 1.85 \\
\hline Put on rope & 1 & & 2.0 & & 2.31 \\
\hline Cut tobacco & 3 & & 2.1 & & 2.43 \\
\hline Sweeping & 7 & & 2.2 & & 2.54 \\
\hline Wash clothes & 3 & & 2.4 & & 2.77 \\
\hline Disbud tobacco & 2 & & 2.4 & & 2.77 \\
\hline Collect cocoa & 1 & & 2.5 & & 2.89 \\
\hline Cut weeds, sarif & 1 & & 2.6 & & 3.01 \\
\hline Collect leaves along the path & 1 & & 2.6 & & 3.01 \\
\hline Dig holes for planting & 2 & & 3.7 & & 4.28 \\
\hline Catch crabs & 1 & & 3.9 & & 4.51 \\
\hline Walking & 26 & & 3.5 & & 4.05 \\
\hline Walking slowly & 10 & & 2.6 & & 3.01 \\
\hline Walking around & 16 & & 1.8 & & 2.08 \\
\hline Walking with load & 19 & & 3.4 & & 3.93 \\
\hline Weeding & 12 & & 2.3 & & 2.66 \\
\hline Clean garden & 4 & & 3.5 & & 4.05 \\
\hline Plant taro & 6 & & 3.1 & & 3.58 \\
\hline Dig taro & 10 & & 2.6 & & 3.01 \\
\hline Cut grass & 5 & & 4.3 & & 4.97 \\
\hline \multicolumn{6}{|l|}{ LUFA men } \\
\hline Lying & 34 & 1.28 & & 1.18 & \\
\hline Sitting & 34 & 1.6 & & 1.48 & \\
\hline Standing & 32 & 1.47 & & 1.36 & \\
\hline \multicolumn{6}{|l|}{ Sitting activities } \\
\hline Make arrows & 5 & 1.8 & & 1.66 & \\
\hline Play 'matches'/cards & 3 & 1.5 & & 1.39 & \\
\hline Weave pitpit wall & 2 & 1.9 & & 1.75 & \\
\hline Unload mumu stone & 1 & & & 1.66 & \\
\hline Sharpen axe & 1 & & & 1.66 & \\
\hline Prepare food (peel tubers) & 1 & 1.4 & & 1.29 & \\
\hline String loom & 1 & 2.0 & & 1.85 & \\
\hline \multicolumn{6}{|l|}{ Standing activities } \\
\hline Pick coffee & 10 & 2.6 & & 2.4 & \\
\hline Chop firewood & 7 & 5.0 & & 4.6 & \\
\hline Collect bush rope & 1 & 4.2 & & 3.88 & \\
\hline Play football in village & 1 & 3.3 & & 3.05 & \\
\hline Walking & 7 & 4.2 & & 3.88 & \\
\hline Walking slowly & 1 & 3.1 & & 2.86 & \\
\hline Walking uphill slowly & 2 & 5.0 & & 4.62 & \\
\hline Walking uphill average & 19 & 6.0 & & 5.54 & \\
\hline Walking uphill fast & 5 & 7.9 & & 7.29 & \\
\hline Walking downhill slowly & 3 & 3.0 & & 2.77 & \\
\hline Walking downhill average & 18 & 3.3 & & 3.05 & \\
\hline Walking downhill fast & 3 & 3.8 & & 3.51 & \\
\hline Walking with load uphill & 3 & 7.1 & & 6.56 & \\
\hline Clearing ground & 6 & 4.9 & & 4.52 & \\
\hline Dig ground & 4 & 5.9 & & 5.45 & \\
\hline Cut pitpit & 1 & 3.1 & & 2.86 & \\
\hline Cut tree & 1 & 5.8 & & 5.36 & \\
\hline Split wood for posts & 5 & 4.4 & & 4.06 & \\
\hline Sharpen posts & 2 & 4.2 & & 3.88 & \\
\hline Put in fence posts & 3 & 4.6 & & 4.25 & \\
\hline Tie fence posts & 4 & 3.3 & & 3.05 & \\
\hline Shovelling road work & 1 & 5.0 & & 4.62 & \\
\hline Dig barat & 1 & 6.5 & & 6.0 & \\
\hline Tie sugar cane & 2 & 3.2 & & 2.95 & \\
\hline Tie banana stem & 2 & 3.5 & & 3.23 & \\
\hline Clean garden & 6 & 4.9 & & 4.52 & \\
\hline Weeding & 5 & 2.6 & & 2.4 & \\
\hline Hunting birds & 1 & 3.6 & & 3.32 & \\
\hline Pull kunai grass & 1 & 2.7 & & 2.49 & \\
\hline Roof house & 1 & 3.1 & & 2.86 & \\
\hline
\end{tabular}


Table 42 Continued

\begin{tabular}{|c|c|c|c|c|}
\hline \multirow[b]{2}{*}{ Activities } & \multirow[b]{2}{*}{$n$} & \multicolumn{2}{|c|}{$\begin{array}{l}\text { Energy cost } \\
\text { of activity } \\
\left(\text { kcal min }{ }^{-1}\right)\end{array}$} & PAR \\
\hline & & Men & Women & Men Women \\
\hline \multicolumn{5}{|l|}{ LUFA women } \\
\hline Lying & 31 & & 1.11 & 1.32 \\
\hline Sitting & 29 & & 1.21 & 1.43 \\
\hline Standing & 30 & & 1.29 & 1.53 \\
\hline \multicolumn{5}{|l|}{ Sitting or squatting activities } \\
\hline Sew clothes & 1 & & 1.3 & 1.54 \\
\hline Skin coffee & 3 & & 1.4 & 1.66 \\
\hline Sew pandanus mat & 2 & & 1.4 & 1.66 \\
\hline Load mumu with food & 1 & & 2.4 & 2.85 \\
\hline Preparing rope & 9 & & 1.3 & 1.54 \\
\hline Weaving bilum & 1 & & 1.4 & 1.66 \\
\hline Peeling sweet potato & 7 & & 1.3 & 1.54 \\
\hline Roasting corn & 1 & & 1.2 & 1.42 \\
\hline Walking & 3 & & 3.3 & 3.91 \\
\hline Walking around & 2 & & 2.4 & 2.85 \\
\hline Walking with load & 1 & & 7.0 & 8.3 \\
\hline Walking uphill slowly & 1 & & 3.6 & 4.27 \\
\hline Walking uphill average & 17 & & 5.1 & 6.05 \\
\hline Walking uphill fast & 2 & & 6.0 & 7.12 \\
\hline Walking downhill slowly & 4 & & 2.1 & 2.49 \\
\hline Walking downhill average & 13 & & 2.7 & 3.2 \\
\hline Walking downhill fast & 5 & & 3.1 & 3.68 \\
\hline Walking with load uphill & 10 & & 5.5 & 6.52 \\
\hline Walking with load downhill & 1 & & 4.2 & 4.98 \\
\hline Clearing ground & 6 & & 3.3 & 3.91 \\
\hline Dig ground & 9 & & 4.2 & 4.98 \\
\hline Weeding & 8 & & 2.6 & 3.08 \\
\hline Plant sweet potato & 3 & & 4.2 & 4.98 \\
\hline Collect sweet potato & 9 & & 2.7 & 3.2 \\
\hline Pick coffee & 10 & & 3.1 & 3.68 \\
\hline
\end{tabular}

Abbreviations: BMR - basal metabolic rate; PAR - physical activity ratio. Subjects: KAUL: 51 males (age 34 years, weight $56 \mathrm{~kg}$, height $160 \mathrm{~cm}$, per cent fat 10\%.) 69 females (age 31 years, weight $47.3 \mathrm{~kg}$, height $151 \mathrm{~cm}$, per cent fat 22\%). LUFA: 43 males (age 28 years, height $159 \mathrm{~cm}$, weight $57.5 \mathrm{~kg}$, per cent fat $10 \%$ ) Women (age 25 years, height $152 \mathrm{~cm}$, weight $50.9 \mathrm{~kg}$, per cent fat $22 \%$ ).

Equipment: lying down and sitting activities measured using Douglas bag and Lloyd Haldane apparatus for $\mathrm{O}_{2}$ and $\mathrm{CO}_{2}$.

For other activities - Max Planck respirometer was used.

PAR calculated from predicted BMR.

Table 43 Oberoi, 1983. Manual and machine washing of clothes $^{46}$

\begin{tabular}{|c|c|c|c|c|}
\hline \multirow[b]{2}{*}{ Activities } & \multicolumn{2}{|c|}{$\begin{array}{c}\text { Energy } \\
\text { cost of activity } \\
\left(\mathrm{kcal} \mathrm{m}^{2} \mathrm{~min}^{-1}\right)\end{array}$} & \multicolumn{2}{|c|}{ PAR } \\
\hline & Men & Women & Men & Women \\
\hline Squatting on the ground & & 1.72 & & 3.0 \\
\hline Sitting on patra $(6.5 \mathrm{~cm}$ stool) & & 1.14 & & 1.99 \\
\hline Sitting on pihri $(13 \mathrm{~cm})$ & & 1.56 & & 2.7 \\
\hline Standing at sink & & 1.15 & & 2.0 \\
\hline Machine wash (part manual) & & 1.10 & & 1.92 \\
\hline
\end{tabular}

Abbreviations: BMR - basal metabolic rate; PAR - physical activity ratio. Subjects: Indian female college students, $n=15$ (age 20.7 years, weight $48.5 \mathrm{~kg}$, height $155.3 \mathrm{~cm}$, all means).

Measurements: expired gas in Douglas bag - Orset volumetric $\mathrm{O}_{2}$. PAR based on predicted BMR.
Table 44 O'Connell, 1986. Energy cost of simulated stair case climbing for firemen (fully geared) ${ }^{47}$

\begin{tabular}{|c|c|c|c|c|}
\hline \multirow[b]{2}{*}{ Activities } & \multicolumn{2}{|c|}{$\begin{array}{c}\text { Energy } \\
\text { cost of activity } \\
\left(\mathrm{VO}_{2} \text { in litre } \min ^{-1}\right)\end{array}$} & \multicolumn{2}{|c|}{ PAR } \\
\hline & Men & Women & Men & Women \\
\hline $\begin{array}{l}\text { Staircase climbing with } \\
\text { turnouts, boots, breathing } \\
\text { apparatus, and } 50 \mathrm{ft}, \\
11 / 2 \text { 'canvas hose with } \\
\text { nozzle and coupling on } \\
\text { shoulder' (wt = } 86.5 \mathrm{lb} \text { ) }\end{array}$ & $\begin{array}{c}3.15 \pm 0.28 \\
\text { mean } \pm \mathrm{SD} \\
\mathrm{VO}_{2}\end{array}$ & & 12.22 & \\
\hline
\end{tabular}

Abbreviations: BMR - basal metabolic rate; PAR - physical activity ratio. Subjects: 17 firefighters, all male (age 32.3 years, height $182.1 \mathrm{~cm}$, weight $82 \mathrm{~kg}$, per cent fat 15.3).

Equipment: breathing apparatus linked to Beckman $\mathrm{O}_{2}$ analyser (OM-11). PAR based on predicted BMR.

Table 45 Pal, 1994. Energy cost of mining activities in India ${ }^{48}$

\begin{tabular}{|c|c|c|c|c|c|}
\hline \multirow[b]{2}{*}{ Activities } & \multirow[b]{2}{*}{$n$} & \multicolumn{2}{|c|}{$\begin{array}{c}\text { Energy } \\
\text { cost of activity } \\
\left(\mathrm{kJ} \mathrm{min}^{-1}\right)\end{array}$} & \multicolumn{2}{|c|}{ PAR } \\
\hline & & Men & Women & Men & Women \\
\hline \multicolumn{6}{|l|}{ Drilling with jackhammer } \\
\hline Tunnel face & 9 & 16.76 & & 3.55 & \\
\hline Post and pillar & 10 & 14.87 & & 3.15 & \\
\hline Horizontal cut and fill & 7 & 19.69 & & 4.17 & \\
\hline Room and pillar & 4 & 22.84 & & 4.84 & \\
\hline \multicolumn{6}{|l|}{ Timbering/grouting } \\
\hline Timbering/preparing face & 5 & 18.22 & & 3.97 & \\
\hline Grouting operation & 7 & 19.06 & & 4.15 & \\
\hline \multicolumn{6}{|l|}{ Loading operation 1} \\
\hline Cavo loader & 12 & 17.18 & & 3.67 & \\
\hline Eimco loader & 12 & 18.65 & & 3.99 & \\
\hline \multicolumn{6}{|l|}{ Loading operation 2} \\
\hline LHD Operator & 4 & 9.43 & & 2.04 & \\
\hline \multicolumn{6}{|l|}{ Manual mining } \\
\hline Shovelling job & 10 & 21.37 & & 4.59 & \\
\hline
\end{tabular}

Abbreviations: BMR - basal metabolic rate; PAR - physical activity ratio. Subjects: 54 male miners. Variable numbers for each activity. Anthropometric characteristics of each subset of subjects provided by the authors. Equipment: Oxylog, $\mathrm{O}_{2}$ converted to energy using $21 \mathrm{~kJ}$ per litre. Measurements: carried at between $28^{\circ} \mathrm{C}$ and $32^{\circ} \mathrm{C}$ and humidity between $50 \%$ and $99 \%$.

PAR based on predicted BMR. 
Table 46 Phillips, 1954. Energy cost of common West African agricultural activities ${ }^{49}$

\begin{tabular}{|c|c|c|c|c|c|}
\hline \multirow[b]{2}{*}{ Activities } & \multirow[b]{2}{*}{$n$} & \multicolumn{2}{|c|}{$\begin{array}{l}\text { Energy cost } \\
\text { of activity } \\
\left(\mathrm{kcal} \mathrm{min}^{-1}\right)\end{array}$} & \multicolumn{2}{|c|}{ PAR } \\
\hline & & Men & Women & Men & Women \\
\hline $\begin{array}{l}\text { Grass cutting: cut as in a sickle with body bent at the waist and } \\
\text { head down }\end{array}$ & 6 & 4.48 & & 4.26 & \\
\hline $\begin{array}{l}\text { Bush clearing: using a machete, } 18 \text { inches long and } 2 \text { inches } \\
\text { wide }\end{array}$ & 6 & 6.2 & & 5.89 & \\
\hline $\begin{array}{l}\text { Hoeing: short spade - for earthing up root crops and } \\
\text { weeding and clearing small root stumps }\end{array}$ & 6 & 4.57 & & 4.34 & \\
\hline \multicolumn{6}{|l|}{$\begin{array}{l}\text { Load carrying using a shallow basin on the head ( } 2 \mathrm{ft} \text { in } \\
\text { diameter and } 6 \text { inches deep). Brick, rubble and cement } \\
\text { is often transported like this }\end{array}$} \\
\hline Head planning $(20 \mathrm{~kg})$ & 6 & 3.63 & & 3.45 & \\
\hline Head planning ( $30 \mathrm{~kg}$ ) & 6 & 4.42 & & 4.2 & \\
\hline Head planning (35 kg) & 5 & 5.25 & & 4.99 & \\
\hline $\begin{array}{l}\text { Log carrying }(20 \mathrm{~kg}) \text { : on the head with one hand up to steady } \\
\text { the log }\end{array}$ & 6 & 3.55 & & 3.37 & \\
\hline Tree felling & 5 & 8.4 & & 7.98 & \\
\hline $\begin{array}{l}\text { Sawing: sawing is away from the body with the blade held in } \\
\text { a vertical position }\end{array}$ & 5 & 6.0 & & 5.7 & \\
\hline Walking & 6 & 3.06 & & 2.91 & \\
\hline Sitting & 7 & 1.3 & & 1.24 & \\
\hline Standing & 6 & 1.3 & & 1.24 & \\
\hline
\end{tabular}

Abbreviations: BMR - basal metabolic rate; PAR - physical activity ratio.

Subjects: Seven Nigerian males. Variable numbers for different activities (age 28.6 years, weight $54.7 \mathrm{~kg}$, height $163.4 \mathrm{~cm}$, all means). Equipment: Douglas bag of 500 I capacity linked to Haldane apparatus.

Measurements: at least two per subject per activity at intervals of 2-3 weeks.

PAR based on predicted BMR.

Table 47 Ramana Murthy, 1966. Energy cost of agricultural activities in India ${ }^{50}$

\begin{tabular}{|c|c|c|c|c|c|}
\hline \multirow[b]{2}{*}{ Activities } & \multirow[b]{2}{*}{$n$} & \multicolumn{2}{|c|}{$\begin{array}{l}\text { Energy cost } \\
\text { of activity } \\
\left(\mathrm{kcal} \mathrm{min}^{-1}\right)\end{array}$} & \multicolumn{2}{|c|}{ PAR } \\
\hline & & Men & Women & Men & Women \\
\hline Ploughing & 11 & 5.48 & & 5.79 & \\
\hline Puddling & 11 & 6.45 & & 6.75 & \\
\hline Working push hoe & 12 & 4.66 & & 4.87 & \\
\hline Trimming bunds & 10 & 6.28 & & 6.73 & \\
\hline $\begin{array}{l}\text { Making channels } \\
\text { for irrigation }\end{array}$ & 6 & 3.25 & & 3.53 & \\
\hline Harvesting & 10 & 3.8 & & 4.08 & \\
\hline Making of bundles & 9 & 3.48 & & 3.68 & \\
\hline Threshing & 9 & 5.27 & & 5.56 & \\
\hline
\end{tabular}

Abbreviations: BMR - basal metabolic rate; PAR - physical activity ratio. Subjects: 30 male labourers who had worked for at least 5 years, variable numbers for each activity. Authors provide anthropometry of each data subset.

Equipment: Kofranyi-Michaelis calorimeter. Gas analysis using a portable Haldane gas analysis apparatus.

PAR calculated using predicted BMR. 
Table 48 Raven, 1973. Energy cost of specific tasks of aluminium smelter workers in Tennessee ${ }^{51}$

\begin{tabular}{|c|c|c|c|c|}
\hline \multirow[b]{2}{*}{ Activities } & \multicolumn{2}{|c|}{$\begin{array}{l}\text { Energy cost of } \\
\text { activity }\left(\mathrm{kcal} \mathrm{min}{ }^{-1}\right)\end{array}$} & \multicolumn{2}{|c|}{ PAR } \\
\hline & Men & Women & Men & Women \\
\hline \multicolumn{5}{|l|}{ Subject 1} \\
\hline Sitting & 1.85 & & 1.48 & \\
\hline Using automatic crowbar on 1 pot & 4.23 & & 3.38 & \\
\hline Oreing pot & 3.75 & & 3.0 & \\
\hline \multicolumn{5}{|l|}{ Subject 2} \\
\hline Sitting & 1.35 & & 1.05 & \\
\hline Using automatic CB on front of pot & 3.05 & & 2.38 & \\
\hline Using $C B$ at front of pot for 2 pots & 3.1 & & 2.42 & \\
\hline Walking sweeping and oreing 2 pots & 3.75 & & 2.93 & \\
\hline On CB for 1 pot & 3.15 & & 2.46 & \\
\hline \multicolumn{5}{|l|}{ Subject 3} \\
\hline Sitting & 2.1 & & 1.63 & \\
\hline Break crust with hand jack hammer & 5.6 & & 4.34 & \\
\hline $\begin{array}{l}\text { Move cradle for carrying hand jackhammer } \\
\text { next to pot and break crust }\end{array}$ & 4.2 & & 3.26 & \\
\hline \multicolumn{5}{|l|}{ Subject 9} \\
\hline Break crust with jackhammer & 6.95 & & 5.43 & \\
\hline Remove cover over pots & 7.1 & & 5.54 & \\
\hline Unhook and hook carbons & 3.5 & & 2.73 & \\
\hline Loosen carbon with pneumatic wrench & 5.15 & & 4.02 & \\
\hline Placing carbon into position & 4.55 & & 3.55 & \\
\hline Set carbon and tighten clamps & 3.75 & & 2.93 & \\
\hline Recovery of molten metal & 4.85 & & 3.79 & \\
\hline Moving siphon & 6.25 & & 4.88 & \\
\hline \multicolumn{5}{|l|}{ Subject 8} \\
\hline Sitting & 1.75 & & 1.4 & \\
\hline Walking & 2.75 & & 2.21 & \\
\hline Rowelling pot & 5.4 & & 4.33 & \\
\hline \multicolumn{5}{|l|}{ Subject 7} \\
\hline Sitting & 1.35 & & 1.07 & \\
\hline Using jackhammer with extension to clean crucible & 5.53 & & 4.39 & \\
\hline Jackhammer mainly held overhead and at chest height & 4.43 & & 3.52 & \\
\hline Using jackhammer overhead resting on crowbar support & 6.35 & & 5.05 & \\
\hline \multicolumn{5}{|l|}{ Subject 10} \\
\hline Cleaned $1-\frac{1}{2}$ Butts using jackhammer & 6.45 & & 4.92 & \\
\hline \multicolumn{5}{|l|}{ Subject 11} \\
\hline Potman riding tricycle across $\frac{1}{2}$ the pot line $\left(\frac{1}{2}\right.$ a room $)$ & 3.25 & & 2.42 & \\
\hline
\end{tabular}

Abbreviations: BMR - basal metabolic rate; PAR - physical activity ratio.

Subjects: Eight subjects - details of age and anthropometry for each subject provided by the authors.

Measurements: 'Spot collection' of expired gases, using a light-weight gas collection system with a manually operated valve linked to Beckman $\mathrm{C}_{2} \mathrm{O}_{2}$ analyser and $\mathrm{CO}_{2}$ absorber. $\mathrm{VO}_{2}$ was recomputed to provide kcal min ${ }^{-1}$.

The jobs ranged from sweeping and light raking of bauxite ore across the pot surface to the wielding of a $14 \mathrm{lb}$ sledgehammer to break up the remnants of a carbon anode into pieces. Generally, hand held jackhammers were used to break the surface crust that was formed on the molten metal during the reduction process. Molten metal was siphoned under vacuum from the reduction pot into a large cylindrical cradle, which was manoeuvred from place to place by an overhead crane. Anodes (carbons) were moved into position by the crane after being hooked to the winch by the worker. Final positioning of the anode within the pot was accomplished by the worker using a crowbar. The collecting cradle was cleaned by breaking the remaining slag from the sides and bottom of the container with a hand-held jackhammer.

PAR based on predicted BMR. Data provided for each subject separately.

Table 49 Samata A, 1981. Energy cost of manual lifting of loads by Indians ${ }^{52}$

\begin{tabular}{|c|c|c|c|c|}
\hline \multirow[b]{2}{*}{ Activities } & \multicolumn{2}{|c|}{$\begin{array}{l}\text { Energy cost of } \\
\text { activity }\left(\mathrm{kJ} \mathrm{min}^{-1}\right)\end{array}$} & \multicolumn{2}{|c|}{ PAR } \\
\hline & Men & Women & Men & Women \\
\hline Load $9 \mathrm{~kg}$, rate 9 per min, ht $1.55 \mathrm{~m}$ & $24.78 / 3.42$ & & 5.78 & \\
\hline Load $16.3 \mathrm{~kg}$, rate 9 per min, ht $1.55 \mathrm{~m}$ & $41.37 / 4.59$ & & 9.65 & \\
\hline
\end{tabular}

Abbreviations: BMR - basal metabolic rate; PAR - physical activity ratio.

Subjects: $n=21$ ? males (age 29.3 years, weight $52.1 \mathrm{~kg}$, height $166 \mathrm{~cm}$, all means).

Equipment: Douglas bag with Haldane Gas analysis apparatus.

Measurements: collection of expired air 10 minutes after start of task.

PAR based on predicted BMR. 
Table 50 Samanta, 1987. Load (head) carrying by physically active, healthy, Indian porters ${ }^{53}$

\begin{tabular}{lclll}
\hline & \multicolumn{2}{c}{$\begin{array}{c}\text { Energy cost of } \\
\text { activity }\left(\mathrm{kJ} \mathrm{min}^{-1}\right)\end{array}$} & & \multicolumn{2}{c}{ PAR } \\
\cline { 2 - 3 } Activities & Men & Women & Men & Women \\
\hline Load $(\mathrm{kg})$ & & & \\
0 & $9.67 / 0.86$ & 2.26 & \\
20 & $14.61 / 1.47$ & 3.42 & \\
30 & $17.9 / 1.58$ & 4.18 & \\
40 & $21.6 / 2.15$ & 5.05 & \\
50 & $26.1 / 2.71$ & 6.1 & \\
60 & $30.0 / 3.83$ & 7.01 & \\
\hline
\end{tabular}

Abbreviations: BMR - basal metabolic rate; PAR - physical activity ratio. Subjects: Five male subjects $(20-29$ years, weight $51.8 \mathrm{~kg}$, height $160.2 \mathrm{~cm})$.

Equipment: Douglas bag with Haldane Gas analysis.

Measurements: for 10 minutes, speed of walking $=5 \mathrm{~km} \mathrm{~h}^{-1}$

PAR based on predicted BMR.
Table 51 Schmidt, 1985. Energy cost of kendo (traditional Japanese fencing $)^{54}$

\begin{tabular}{|c|c|c|c|c|}
\hline \multirow[b]{2}{*}{ Activities } & \multicolumn{2}{|c|}{$\begin{array}{l}\text { Energy cost } \\
\text { of activity } \\
\left(\mathrm{kcal} \mathrm{min}^{-1}\right)\end{array}$} & \multicolumn{2}{|c|}{ PAR } \\
\hline & Men & Women & Men & Women \\
\hline $\begin{array}{l}5 \text { min Kendo bout } \\
\text { wearing full armour }\end{array}$ & 15.64 & & 12.97 & \\
\hline
\end{tabular}

Abbreviations: BMR - basal metabolic rate; PAR - physical activity ratio. Subjects: Eight adult trained ( $>5$ years), male, Caucasian kendokas (age 28.4 years, height $176.8 \mathrm{~cm}$, weight $69.3 \mathrm{~kg}$, per cent fat $14.4 \%$ ).

Equipment: open-circuit gas analysis - Parkinson-Cowan dry gas meter and a Beckman gas analyser.

PAR calculated from predicted BMR.

Table 52 Sheldahl, 1992. The energy cost of shovelling snow ${ }^{55}$

\begin{tabular}{llllll}
\hline & \multicolumn{2}{c}{$\begin{array}{l}\text { Energy cost of activity } \\
\left(\mathrm{VO}_{2}-\mathrm{ml} \mathrm{kg}^{-1} \mathrm{~min}^{-1}\right)\end{array}$} & & \multicolumn{2}{c}{ PAR } \\
\cline { 2 - 3 } \cline { 5 - 6 } Activities & Men & Women & & Men & Women \\
\hline Self-paced lift-throw & 25.2 & & 8.08 & \\
Self-paced push-throw & 24.4 & & 7.82 & \\
Paced lift-throw & 24.0 & & 7.7 & \\
\hline
\end{tabular}

Abbreviations: BMR - basal metabolic rate; PAR - physical activity ratio.

Subjects: 12 younger normal men (age 40 years, weight $81 \mathrm{~kg}$, all means).

Equipment and measurement: open-circuit spirometry. $\mathrm{VO}_{2}$ determined at 6 minutes and 8 minutes of each procedure.

Analysis of $\mathrm{O}_{2}$ done within 5 minutes of collection. Snow depth of 3-4 inches

PAR calculated from predicted BMR.

Table 53 Spurr, 1975. Energy cost of cutting sugar cane ${ }^{56}$

\begin{tabular}{|c|c|c|c|c|}
\hline \multirow[b]{2}{*}{ Activities } & \multicolumn{2}{|c|}{$\begin{array}{l}\text { Energy cost of activity } \\
\left(\mathrm{VO}_{2}-\text { litre } \mathrm{min}^{-1}\right)\end{array}$} & \multicolumn{2}{|c|}{ PAR } \\
\hline & Men & Women & Men & Women \\
\hline \multicolumn{5}{|l|}{ am } \\
\hline Rest-sitting position & 0.25 & & 1.15 & \\
\hline $5-10$ min after starting sugar cane cutting & 1.50 & & 6.88 & \\
\hline $20-25$ min after starting cutting sugar cane & 1.46 & & 6.7 & \\
\hline \multicolumn{5}{|l|}{ pm } \\
\hline Rest-sitting position & 0.25 & & 1.15 & \\
\hline 5-10 min after starting sugar cane cutting & 1.51 & & 6.9 & \\
\hline $20-25$ min after starting cutting sugar cane & 1.44 & & 6.6 & \\
\hline
\end{tabular}

Abbreviations: BMR - basal metabolic rate; PAR - physical activity ratio.

Subjects: 61 ? males who were experienced sugar cane cutters (age 29.8 years, height $163.3 \mathrm{~cm}$, weight $58.6 \mathrm{~kg}$, per cent fat $10.3 \%$, all means)

Equipment: Kofranyi-Michaelis respirometer. Expired air analysed using gas chromatography.

PAR calculated from predicted BMR. 
Table 54 Spurr, 1977. Loading sugar cane on to wagons by picking cane singly or in bundles $(1-2 \mathrm{~kg})$ and throwing them onto the wagon $^{57}$

\begin{tabular}{llllll}
\hline & \multicolumn{2}{c}{$\begin{array}{c}\text { Energy cost } \\
\text { of activity } \\
\left(\mathrm{VO}_{2}-{\left.\text { litre } \mathrm{min}^{-1}\right)}\right.\end{array}$} & & & \\
\cline { 2 - 3 } \cline { 5 - 6 } Activities & Men & Women & & Men & Women \\
\hline $\begin{array}{l}\text { Rest for 10 min } \\
\text { prior to loading } \\
\text { Loading }\end{array}$ & 0.25 & & & 1.12 & \\
\hline
\end{tabular}

Abbreviations: BMR - basal metabolic rate; PAR - physical activity ratio. Subjects: 28 men employed as loaders (age 34 years, height $165 \mathrm{~cm}$ weight $60.3 \mathrm{~kg}$, per cent fat $9.9 \%$, all mean).

Equipment/measurements: Kofranyi-Michaelis respirometer - gas concentrations in expired air using gas chromatography. Measurements made in 3,5 minute periods $(10-15,25-30$ and $40-45$ minutes after starting work).

PAR from predicted BMR.

Table 55 Thornton, 1984. Energy cost of helicopter pilots flying two different types of helicopters ${ }^{58}$

\begin{tabular}{|c|c|c|c|c|}
\hline \multirow[b]{2}{*}{ Activities } & \multicolumn{2}{|c|}{$\begin{array}{l}\text { Energy cost of } \\
\text { activity (watts) }\end{array}$} & \multicolumn{2}{|c|}{ PAR } \\
\hline & Men & Women & Men & Women \\
\hline \multicolumn{5}{|c|}{ Gazelle (light observation helicopter) } \\
\hline Hover $n=6$ & 145 & & 1.67 & \\
\hline Level flight (1000 ft) & 128 & & 1.47 & \\
\hline \multicolumn{5}{|c|}{ Puma (medium battlefield support helicopter) } \\
\hline Hover $n=3$ & 232 & & 2.59 & \\
\hline $\begin{array}{l}\text { Level flight } \\
\qquad(1000 \mathrm{ft}) n=6\end{array}$ & 197 & & 2.19 & \\
\hline
\end{tabular}

Abbreviations: BMR - basal metabolic rate; PAR - physical activity ratio. Subjects: 12 trained male defence pilots, under peace-time conditions, divided into two groups. Anthropometry details provided in the paper.

Equipment: Oxylog.

PAR calculated using predicted BMR.

Table 56 Tin-May-Than, 1988. Energy cost of weaving and domestic chores in Burmese ${ }^{59}$

\begin{tabular}{|c|c|c|c|c|c|}
\hline \multirow[b]{2}{*}{ Activities } & \multirow[b]{2}{*}{$n$} & \multicolumn{2}{|c|}{$\begin{array}{l}\text { Energy cost } \\
\text { of activity } \\
\left(\mathrm{kcal} \mathrm{min}^{-1}\right)\end{array}$} & \multicolumn{2}{|c|}{ PAR } \\
\hline & & Men & Women & Men & Women \\
\hline Weaving & 19 & & 2.59 & & 3.19 \\
\hline Spinning (sitting) & 2 & & 1.91 & & 2.29 \\
\hline Spinning (standing) & 1 & & 1.52 & & 1.89 \\
\hline $\begin{array}{l}\text { Picking thread } \\
\text { (sitting) }\end{array}$ & 3 & & 1.32 & & 1.662 \\
\hline $\begin{array}{l}\text { 'Making paste' } \\
\text { Burmese makeup }\end{array}$ & 6 & & 2.35 & & 2.94 \\
\hline Washing clothes & 2 & & 2.2 & & 2.5 \\
\hline Sitting (leisure) & 15 & & 1.14 & & 1.4 \\
\hline Walking & 18 & & 2.22 & & 2.75 \\
\hline
\end{tabular}

Abbreviations: BMR - basal metabolic rate; PAR - physical activity ratio Subjects: $n=22$. Variable numbers for different activities (age 19-43 years, height $150.8 \mathrm{~cm}$, weight $45.9 \mathrm{~kg}$, per cent fat 25.8 , all means). Measurements: Douglas bag collections, volume and expired gas analysis. PAR based on predicted BMR.
Table 57 Torun, 1982. Energy costs of agricultural and domestic chores $^{60}$

\begin{tabular}{|c|c|c|c|c|c|}
\hline \multirow[b]{2}{*}{ Activities } & \multirow[b]{2}{*}{$n$} & \multicolumn{2}{|c|}{$\begin{array}{l}\text { Energy cost } \\
\text { of activity } \\
\left(\mathrm{kcal} \mathrm{min}^{-1}\right)\end{array}$} & \multicolumn{2}{|c|}{ PAR } \\
\hline & & Men & Women & Men & Women \\
\hline Lying down & 23 & & 1.14 & & 1.3 \\
\hline Standing & 8 & & 1.15 & & 1.31 \\
\hline Sitting/sewing & 20 & & 1.2 & & 1.36 \\
\hline Ironing clothes & 1 & & 1.44 & & 1.66 \\
\hline Picking coffee & 6 & & 1.5 & & 1.7 \\
\hline $\begin{array}{l}\text { Winnowing or } \\
\text { dekernelising corn }\end{array}$ & 15 & & 1.63 & & 1.85 \\
\hline Washing dishes & 1 & & 1.68 & & 1.91 \\
\hline Cooking & 19 & & 1.75 & & 1.99 \\
\hline Making Tortillas & 48 & & 2.08 & & 2.36 \\
\hline House cleaning & 16 & & 2.2 & & 2.5 \\
\hline Child care & 4 & & 2.22 & & 2.52 \\
\hline Washing clothes & 16 & & 2.69 & & 3.06 \\
\hline $\begin{array}{l}\text { Walking on a flat terrain } \\
\text { without a load }\end{array}$ & 31 & & 2.73 & & 3.1 \\
\hline $\begin{array}{l}\text { Walking on a flat terrain } \\
\text { carrying } 5 \mathrm{~kg}\end{array}$ & & & 2.98 & & 3.39 \\
\hline $\begin{array}{l}\text { Walking on a flat terrain } \\
\text { carrying } 10 \mathrm{~kg}\end{array}$ & & & 3.22 & & 3.65 \\
\hline Sweeping & 33 & & 3.12 & & 3.55 \\
\hline Cutting fruit with a pole & 1 & & 3.34 & & 3.8 \\
\hline Gleaning & 5 & & 3.95 & & 4.49 \\
\hline $\begin{array}{l}\text { Lifting and moving } \\
\text { objects }\end{array}$ & 4 & & 4.04 & & 4.59 \\
\hline Walking uphill & 18 & & 4.25 & & 4.83 \\
\hline Chopping wood & & & 4.32 & & 4.91 \\
\hline $\begin{array}{l}\text { Carrying a } 10 \mathrm{~kg} \\
\text { load uphill }\end{array}$ & 24 & & 5.77 & & 6.56 \\
\hline
\end{tabular}

Abbreviations: BMR - basal metabolic rate; PAR - physical activity ratio. Subjects: $n=58$, Guatamelan rural women, 12 in 2nd or 3rd trimester, 30 lactating (age 27 years, weight $49.1 \mathrm{~kg}$, height $150 \mathrm{~cm}$, all means).

Equipment: Kofranyi-Michaelis initially, but most with Douglas bag and subsequent calibrated analysis.

Measurements: collections were for $2-5$ minutes, starting after 2 minutes for light tasks and 3-5 minutes for heavy tasks.

PAR calculated using predicted BMR.

Table 58 Town, 1980. Energy cost of rope skipping in both men and women ${ }^{61}$

\begin{tabular}{|c|c|c|c|c|}
\hline \multirow[b]{2}{*}{ Activities } & \multicolumn{2}{|c|}{$\begin{array}{l}\text { Energy cost } \\
\text { of activity } \\
\left(\mathrm{kcal} \mathrm{min}^{-1}\right)\end{array}$} & \multicolumn{2}{|c|}{ PAR } \\
\hline & Men & Women & Men & Women \\
\hline 125 skips per min & 15.7 & 10.9 & 12.5 & 12.27 \\
\hline 135 skips per min & 16.0 & 11.1 & 12.75 & 12.49 \\
\hline 145 skips per min & 16.5 & 10.9 & 13.15 & 12.26 \\
\hline
\end{tabular}

Abbreviations: BMR - basal metabolic rate; PAR - physical activity ratio. Subjects: 11 females (age 21.8 years, weight $53.3 \mathrm{~kg}$ ) and 19 males $(24.7$ years, weight $73.7 \mathrm{~kg}$ ).

Equipment: modified gas collecting system to allow skipping. Gas collected via tubing into meteorological balloons and analysed using Beckman E2 and LB1 analysers.

Measurement: gas collection for 5 minute after start.

PAR calculated using the predicted BMR. 
Table 59 Viteri, 1971. Energy cost of common agricultural activities in Central America ${ }^{62}$

\begin{tabular}{|c|c|c|c|c|c|}
\hline \multirow[b]{2}{*}{ Activities } & \multirow[b]{2}{*}{$n$} & \multicolumn{2}{|c|}{$\begin{array}{l}\text { Energy cost of } \\
\text { activity }\left(\mathrm{kcal} \mathrm{min}{ }^{-1}\right)\end{array}$} & \multicolumn{2}{|c|}{ PAR } \\
\hline & & Men & Women & Men & Women \\
\hline Sitting & 19 & 1.21 & & 1.08 & \\
\hline Standing & 18 & 1.28 & & 1.14 & \\
\hline Office work, wash stable with water hose & 5 & 1.73 & & 1.54 & \\
\hline $\begin{array}{l}\text { Wire fence, sharpen tools, tie iron rods in } \\
\text { construction work, drive truck or tractor, } \\
\text { wash buckets }\end{array}$ & 21 & 2.59 & & 2.31 & \\
\hline $\begin{array}{l}\text { Hackle barn floors, Open holes with a straight } \\
\text { hoe, milk cows }\end{array}$ & 19 & 3.44 & & 3.07 & \\
\hline $\begin{array}{l}\text { Walk with or without moderate load, drive } \\
\text { cattle, shovel hay with a trident, ride } \\
\text { horse, hand mix cattle feed }\end{array}$ & 31 & 4.47 & & 3.99 & \\
\hline $\begin{array}{l}\text { Walk with a heavy load, Push a wheel barrow, } \\
\text { Open ditch with a hoe, shovel sand, Mow with } \\
\text { machete (standing), Cut wood with machete, } \\
\text { cut wood with hand saw }\end{array}$ & 40 & 5.6 & & 5.0 & \\
\hline $\begin{array}{l}\text { Distribute gravel with hoe, hand gather cut weeds, } \\
\text { mow with scythe, mow with machete (leaning), } \\
\text { open deep furrow with hoe or pick, hoe } \\
\text { in water ditch }\end{array}$ & 46 & 6.33 & & 5.65 & \\
\hline $\begin{array}{l}\text { Mow with sickle, shovel heavy material, harvest } \\
\text { forages with machete }\end{array}$ & 12 & 7.18 & & 5.65 & \\
\hline Ride bicycle on farm roads & 6 & 10.0 & & 6.41 & \\
\hline
\end{tabular}

Abbreviations: BMR - basal metabolic rate; PAR - physical activity ratio.

Subjects: 18 men (age 29.7 years, height $160.9 \mathrm{~cm}$, weight $60.1 \mathrm{~kg}$ ).

Equipment: KM respirometer calib at 2-week intervals. Air analysed using the Scholander

Measurements: expired air collected for last 10 minutes of 15-20 minute activity period. Each activity measured in duplicate and the mean taken.

BMR measured in 15 subjects.

Table 60 Wilke, 1995. Variety of household tasks ${ }^{63}$

\begin{tabular}{|c|c|c|c|c|}
\hline \multirow[b]{2}{*}{ Activities } & \multicolumn{2}{|c|}{$\begin{array}{c}\text { Energy cost } \\
\text { of activity } \\
\left(\mathrm{VO}_{2}-\mathrm{ml} \mathrm{kg}^{-1} \mathrm{~min}^{-1}\right)\end{array}$} & \multicolumn{2}{|c|}{ PAR } \\
\hline & Men & Women & Men & Women \\
\hline Vacuum carpet & & $10.7 / 0.9 \mathrm{SE}$ & & 3.88 \\
\hline Mop floor & & $12.2 / 0.7 \mathrm{SE}$ & & 4.43 \\
\hline Change bed & & $12.7 / 0.6 \mathrm{SE}$ & & 4.61 \\
\hline Wash floor & & $13.7 / 0.8 \mathrm{SE}$ & & 4.96 \\
\hline
\end{tabular}

Abbreviations: BMR - basal metabolic rate; PAR - physical activity ratio. Subjects: data presented here only for the normal controls, 10 Caucasian women (age 62 years, weight $64 \mathrm{~kg}$ ).

Measurements: $\min -\max \mathrm{VO}_{2}$ measured by open-circuit spirometry. Each task $6-8$ minute steady state expected to be achieved by 6 minutes PAR based on predicted BMR.
Table 61 Wilmore, 1978. Energy cost of circuit-training (three sets through a 10 station circuit) ${ }^{64}$

\begin{tabular}{|c|c|c|c|c|}
\hline \multirow{2}{*}{ Activities } & \multicolumn{2}{|c|}{$\begin{array}{l}\text { Energy cost } \\
\text { of activity } \\
\left(\mathrm{kcal} \mathrm{min}^{-1}\right)\end{array}$} & \multicolumn{2}{|c|}{ PAR } \\
\hline & Men & Women & Men & Women \\
\hline $\begin{array}{l}\text { Energy cost of } \\
\text { circuit training }\end{array}$ & 9.0 & 6.1 & 6.96 & 6.29 \\
\hline
\end{tabular}

Abbreviations: BMR - basal metabolic rate; PAR - physical activity ratio. Subjects: 20 men (age 23.7 years, height $180.6 \mathrm{~cm}$, weight $77.5 \mathrm{~kg}$, per cent fat 14.3) and 20 women (age 20.3 years, height $165.4 \mathrm{~cm}$, weight $61.0 \mathrm{~kg}$, per cent fat 26.4). All subjects were familiar with the concept and most had been participating in a training programme.

Equipment/measurement: Beckman metabolic measurement cart. Measurements at 45 second intervals for each station through three complete circuits.

PAR calculated using predicted BMR.

Table 62 Zhuo, 1984. Energy cost of Tai-Chi Chuan exercise ${ }^{65}$

\begin{tabular}{|c|c|c|c|c|}
\hline \multirow[b]{2}{*}{ Activities } & \multicolumn{2}{|c|}{$\begin{array}{l}\text { Energy cost of activity } \\
\quad\left(\mathrm{VO}_{2} \text {-litre } \mathrm{min}^{-1}\right)\end{array}$} & \multicolumn{2}{|c|}{ PAR } \\
\hline & Men & Women & Men & Women \\
\hline $\begin{array}{l}\text { The long form of Yang's style with } 108 \text { successive } \\
\text { movements in a set routine. Time req }=17.5-25 \mathrm{~min} \\
\text { (average } 22 \mathrm{~min} \text { ) }\end{array}$ & 1.03 & & 4.19 & \\
\hline
\end{tabular}

Abbreviations: BMR - basal metabolic rate; PAR - physical activity ratio.

Subjects: 11 healthy males who had been practising Tai Chi regularly for 3-8 years (age 28.4 years, height $176.9 \mathrm{~cm}$, weight $71.5 \mathrm{~kg}$, per cent fat 13.9).

Equipment: automated respiratory gas collection system (Jaeger Ergo-Oxyscreen) with paramagnetic and infrared analysers.

PAR calculated using predicted BMR. 
Table 63 Energy costs of a variety of activities abstracted from Passmore and Durnin, $1955^{2}$. Different rows with similar activity identifiers indicate different studies

\begin{tabular}{|c|c|c|c|}
\hline Activities & $n$ & $\begin{array}{l}\text { Energy cost } \\
\left(\mathrm{kcal} \mathrm{min}^{-1}\right)\end{array}$ & PAR \\
\hline \multicolumn{4}{|l|}{ Personal necessities } \\
\hline \multicolumn{4}{|l|}{ Males } \\
\hline Washing hands and face and brushing hair & 1 & 2.5 & 2.3 \\
\hline Washing and dressing & 1 & 2.6 & 2.18 \\
\hline Dressing, washing and shaving & 5 & 3.8 & 3.04 \\
\hline Dressing & 9 & 4.0 & 3.32 \\
\hline Washing and shaving & 4 & 2.6 & 2.3 \\
\hline Dressing & 6 & 3.0 & 2.65 \\
\hline \multicolumn{4}{|l|}{ Females } \\
\hline Washing, dressing and undressing & 3 & 3.3 & 3.3 \\
\hline \multicolumn{4}{|l|}{ Light indoor recreation } \\
\hline \multicolumn{4}{|l|}{ Males } \\
\hline Sitting, listening to the radio & 1 & 2.0 & 1.51 \\
\hline Sitting, listening to the radio & 1 & 2.5 & 1.86 \\
\hline Sitting, writing & 1 & 1.9 & 1.65 \\
\hline Sitting, writing & 1 & 2.2 & 1.64 \\
\hline Sitting playing cards & 1 & 1.9 & 1.44 \\
\hline Sitting playing cards & 1 & 2.1 & 1.64 \\
\hline Sitting, playing accordion & 1 & 2.2 & 1.84 \\
\hline Sitting, playing piano & 1 & 2.5 & 2.25 \\
\hline Sitting, playing cello & 1 & 2.6 & 2.28 \\
\hline Sitting, playing drums & 1 & 4.0 & 3.71 \\
\hline Standing, drawing & 1 & 2.3 & 2.0 \\
\hline Standing, conducting orchestra & 1 & 2.5 & 2.22 \\
\hline Standing playing trumpet & 1 & 2.1 & 1.77 \\
\hline Standing, playing double bass & 1 & 2.5 & 2.49 \\
\hline Playing with children & 3 & 3.5 & 3.11 \\
\hline \multicolumn{4}{|l|}{ Females } \\
\hline Sitting, eating & 1 & 1.5 & 1.55 \\
\hline \multicolumn{4}{|c|}{ Recreations involving moderate exercise (only males) } \\
\hline Driving a car & 3 & 2.8 & 2.43 \\
\hline Driving a motorcycle & 3 & 3.4 & 2.95 \\
\hline Cycling (own pace) & 10 & 8.2 & 6.8 \\
\hline Cycling (own pace) & 1 & 5.9 & 5.48 \\
\hline Cycling (own pace) & 1 & 6.6 & 5.62 \\
\hline Cycling (own pace) & 1 & 10.3 & 8.63 \\
\hline Dancing, petronella & 1 & 4.7 & 3.9 \\
\hline Dancing, foxtrot & 1 & 5.2 & 3.93 \\
\hline Dancing, waltz & 1 & 5.7 & 4.46 \\
\hline Dancing, rumba & 1 & 7.0 & 5.81 \\
\hline Dancing, eightsome reel & 3 & 7.7 & 5.97 \\
\hline Gardening, weeding & 1 & 4.4 & 5.32 \\
\hline Gardening, weeding & 1 & 5.6 & 4.94 \\
\hline Gardening, digging & 1 & 8.6 & 7.69 \\
\hline Gymnastics-balancing exercises & & 2.5 & 2.09 \\
\hline Abdominal exercises & & 3.0 & 2.51 \\
\hline Trunk bending & & 3.5 & 2.93 \\
\hline Arms swinging, hopping & & 6.5 & 5.44 \\
\hline Bowls & 1 & 4.4 & 3.74 \\
\hline Golf & 1 & 5.0 & 4.38 \\
\hline Archery & 2 & 5.2 & 4.69 \\
\hline Cricket, fielding & 6 & 3.9 & 3.15 \\
\hline Cricket, bowling & 6 & 5.2 & 4.21 \\
\hline Cricket, batting & 6 & 6.0 & 4.85 \\
\hline Tennis & 7 & 7.1 & 5.84 \\
\hline \multicolumn{4}{|l|}{ Recreations involving hard exercise } \\
\hline Football, association & & 8.9 & 7.45 \\
\hline Sculling at $51 \mathrm{~m} \mathrm{~min}^{-1}$ & & 4.1 & 3.5 \\
\hline Sculling at $69 \mathrm{~m} \mathrm{~min}^{-1}$ & & 6.4 & 5.46 \\
\hline Sculling at $97 \mathrm{~m} \mathrm{~min}^{-1}$ & & 11.2 & 9.55 \\
\hline Sculling at 61 per min & & 4.8 & 4.36 \\
\hline Sculling at $87 \mathrm{~m} \mathrm{~min}^{-1}$ & & 7.0 & 6.35 \\
\hline Sculling at $93 \mathrm{~m} \mathrm{~min}^{-1}$ & & 9.2 & 9.08 \\
\hline Sculling at $68 \mathrm{~m} \mathrm{~min}^{-1}$ & & 5.5 & 5.34 \\
\hline Swimming, breast stroke & & 11.0 & 9.21 \\
\hline Swimming, back crawl & & 11.5 & 9.63 \\
\hline Playing squash racquets & & 10.2 & 8.62 \\
\hline Cross country running & & 10.6 & 9.12 \\
\hline
\end{tabular}


Table 63 Continued

\begin{tabular}{|c|c|c|c|}
\hline Activities & $n$ & $\begin{array}{l}\text { Energy cost } \\
\left(\mathrm{kcal}^{2} \mathrm{~min}^{-1}\right)\end{array}$ & PAR \\
\hline \multicolumn{4}{|l|}{ Domestic work } \\
\hline \multicolumn{4}{|l|}{ Females } \\
\hline Sewing, 30 stiches a min & 2 & 1.14 & 1.33 \\
\hline Knitting, 23 stiches a min & 2 & 1.17 & 1.37 \\
\hline Sweeping floors & 4 & 1.7 & 1.99 \\
\hline Simple work, sitting & & 1.7 & 1.57 \\
\hline Washing small clothes & & 2.3 & 2.12 \\
\hline Stirring & & 3.0 & 2.77 \\
\hline Bringing in the wash & & 3.3 & 3.05 \\
\hline Polishing floor & & 4.8 & 4.43 \\
\hline Taking and hanging out the washing & & 5.0 & 4.62 \\
\hline Clearing floor, kneeling and bending & & 5.9 & 5.4 \\
\hline Scrubbing & & 7.0 & 6.46 \\
\hline Putting washing through mangle & & 8.0 & 7.39 \\
\hline Scrubbing, standing & 3 & 2.9 & 3.35 \\
\hline Washing small clothes & & 3.0 & 3.1 \\
\hline Kneading dough & & 3.3 & 3.41 \\
\hline Wringing the wash by hand & & 4.4 & 4.54 \\
\hline Beating carpets and mats & & 4.9 & 5.06 \\
\hline Putting washing through a mangle (all) & 1 & 6.0 & 6.2 \\
\hline Scrubbing, kneeling & 3 & 3.4 & \\
\hline Scrubbing floors & & 3.6 & 3.4 \\
\hline Mopping & & 4.2 & 4.0 \\
\hline Taking out and hanging the wash & & 4.5 & 4.25 \\
\hline Bed making and bed stripping & & 5.4 & 5.1 \\
\hline Beating and brushing carpets (all) & 1 & 7.8 & 7.37 \\
\hline \multicolumn{4}{|l|}{ Males } \\
\hline Brushing boots & & 2.2 & 2.02 \\
\hline Cleaning windows (all) & 1 & 3.0 & 2.76 \\
\hline Polishing & 5 & 2.4 & 2.21 \\
\hline Peeling potatoes & 1 & 2.9 & 2.4 \\
\hline Getting in coals & & 3.5 & 2.93 \\
\hline Breaking firewood (all) & 1 & 4.9 & 4.1 \\
\hline Cleaning windows & & 3.7 & 3.3 \\
\hline Tidying beds (all) & 10 & 3.9 & 3.48 \\
\hline Ironing & 5 & 4.2 & 3.55 \\
\hline \multicolumn{4}{|l|}{ Energy cost of light occupational activities } \\
\hline Shoemaker: fixing soles & & $2.4,2.1$ & 2.37 \\
\hline Filling soles & & 2.3 & 2.42 \\
\hline Polishing shoes (all) & 1 & 1.8 & 1.9 \\
\hline Locksmith: filing with large file & & $3.3,3.7$ & 3.38 \\
\hline Five other processes (all) & 1 & $2.1-2.9$ & 2.42 \\
\hline Tailor: cutting & & 2.4 & 2.11 \\
\hline Machine sewing & & $2.8,2.9$ & 2.51 \\
\hline Hand sewing & & 1.9 & 1.67 \\
\hline Pressing (all) & 1 & 3.5 & 3.08 \\
\hline Tailor: cutting & & 2.7 & 2.4 \\
\hline Machine sewing & & $2.6,2.7$ & 2.36 \\
\hline Hand sewing & & 2.0 & 1.78 \\
\hline Pressing & & 4.3 & 3.82 \\
\hline \multicolumn{4}{|c|}{ Energy cost of postmen climbing stairs at usual pace } \\
\hline Load $11 \mathrm{~kg}$ & & 9.8 & 7.71 \\
\hline Load $16 \mathrm{~kg}$ & & 11.5 & 8.88 \\
\hline Load $16 \mathrm{~kg}$ & & 9.8 & 8.21 \\
\hline Load $16 \mathrm{~kg}$ (all) & 1 & 13.8 & 10.7 \\
\hline \multicolumn{4}{|l|}{ Energy expenditure in the building industry } \\
\hline Measuring wood & & 2.4 & 2.16 \\
\hline Machine sawing & & 2.4 & 2.16 \\
\hline Measuring and sawing & & 3.5 & 3.15 \\
\hline Joining floor boards & & 4.4 & 3.96 \\
\hline Miscellaneous work & & 4.5 & 4.05 \\
\hline Drilling hardwood & & 7.0 & 6.31 \\
\hline Chiselling & & 5.7 & 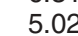 \\
\hline Sawing softwood & & 6.3 & 5.56 \\
\hline Sawing hardwood & & 7.5 & 6.61 \\
\hline Planing softwood & & 8.1 & 7.14 \\
\hline Planing hardwood & & 9.1 & 8.02 \\
\hline
\end{tabular}


Table 63 Continued

\begin{tabular}{|c|c|c|}
\hline Activities & $\begin{array}{l}\text { Energy cost } \\
\left(\mathrm{kcalmin}^{-1}\right)\end{array}$ & PAR \\
\hline \multicolumn{3}{|l|}{ Energy expenditure in agriculture, Russia 1933} \\
\hline \multicolumn{3}{|l|}{ Males } \\
\hline Ploughing & 6.9 & 5.94 \\
\hline Thrashing rye & 5.0 & 4.3 \\
\hline Ploughing & 5.4 & 4.56 \\
\hline Thrashing rye & 4.5 & 3.68 \\
\hline \multicolumn{3}{|l|}{ Females } \\
\hline Binding oats & 3.3 & 3.34 \\
\hline Binding rye & 4.2 & 4.25 \\
\hline Binding oats & 4.1 & 4.68 \\
\hline Binding rye & 4.7 & 5.37 \\
\hline Weeding rape & 3.3 & 3.77 \\
\hline \multicolumn{3}{|c|}{ Energy expenditure in agriculture, the Gambia, 1953} \\
\hline \multicolumn{3}{|c|}{ Males } \\
\hline Clearing shrub and dry grass & 7.1 & 6.35 \\
\hline Ridging (deep digging) & $9.5(5.5-15.2)$ & 8.88 \\
\hline Planting groundnuts & $3.7(3.1-4.5)$ & 3.13 \\
\hline Weeding & $5.3(3.8-7.8)$ & 4.65 \\
\hline \multicolumn{3}{|l|}{ Females } \\
\hline Hoeing & $5.8(4.8-6.8)$ & 6.48 \\
\hline Pounding rice & $5.0(3.9-6.4)$ & 5.4 \\
\hline \multicolumn{3}{|l|}{ Energy expenditure in agriculture, Germany 1953} \\
\hline Milking by hand & 4.7 & 4.08 \\
\hline Machine milking 1 pail & 3.4 & 2.95 \\
\hline Machine milking 2 pails & 3.9 & 3.39 \\
\hline Cleaning milk pails & 4.4 & 3.82 \\
\hline Horse ploughing & 5.9 & 4.79 \\
\hline Horse ploughing (another kind of plough) & 5.1 & 4.14 \\
\hline Tractor ploughing & 4.2 & 3.41 \\
\hline \multicolumn{3}{|l|}{ Energy expenditure working with an axe } \\
\hline \multicolumn{3}{|l|}{ Perpendicular blows } \\
\hline Wt of axe $0.65 \mathrm{~kg}$ & 11.4 & 8.49 \\
\hline \multicolumn{3}{|l|}{ Speed of blows 36 per min } \\
\hline Wt 1.25 & 11.9 & 8.86 \\
\hline \multicolumn{3}{|l|}{ Speed 34 per min } \\
\hline 19 & 6.9 & 5.14 \\
\hline 35 & 11.0 & 8.19 \\
\hline 51 & 24.1 & 17.95 \\
\hline \multirow{2}{*}{\multicolumn{3}{|c|}{ Speed 33 per min }} \\
\hline & & \\
\hline \multicolumn{3}{|l|}{ Horizontal blows } \\
\hline Wt 0.65 & 12.0 & 8.94 \\
\hline Speed 34 per $\min$ & & \\
\hline Wt $1.25 \mathrm{~kg}$ & 13.2 & 9.83 \\
\hline Speed 34 per min & & \\
\hline Wt 2.0 & 12.3 & 9.16 \\
\hline Speed 33 per min & & \\
\hline Energy expenditure of Bristish soldiers & & \\
\hline Anti-gas drill & 2.0 & 1.97 \\
\hline Weapon training & 2.2 & 1.81 \\
\hline Kit inspection & 2.3 & 1.83 \\
\hline Arms drill & 2.4 & 2.01 \\
\hline Musketry & 2.7 & 2.34 \\
\hline Polishing equipment & 2.7 & 2.2 \\
\hline Mixed sports in the gym (incl rest pauses) & 2.7 & 2.17 \\
\hline Cleaning kit and rifle & 2.7 & 2.3 \\
\hline Throwing hand grenade & 2.9 & 2.37 \\
\hline Guard and sentry drill & 3.2 & 2.44 \\
\hline Company drill & 3.4 & 3.13 \\
\hline Squad and platoon drill & 3.7 & 3.07 \\
\hline Mixed sports outdoors (incl rest pauses) & 3.8 & 3.21 \\
\hline Slow march & 3.8 & 3.18 \\
\hline Marching $\left(2 \mathrm{~m} \mathrm{~h}^{-1}\right)$ with $27 \mathrm{~kg}$ load & 3.9 & 3.27 \\
\hline Bayonet exercise & 3.9 & 3.18 \\
\hline Physical exercises & 4.1 & 3.43 \\
\hline Doing fatigues & 4.1 & 3.29 \\
\hline Ironing equipment & 4.2 & 3.55 \\
\hline Drill & 5.0 & 4.11 \\
\hline
\end{tabular}


Table 63 Continued

\begin{tabular}{|c|c|c|c|}
\hline Activities & $n$ & $\begin{array}{l}\text { Energy cost } \\
\left(\mathrm{kcal} \mathrm{min}^{-1}\right)\end{array}$ & PAR \\
\hline Marching $\left(3 \mathrm{~m} \mathrm{~h}^{-1}\right)$ with $27 \mathrm{~kg}$ load & & 5.3 & 4.44 \\
\hline Quick march & & 5.6 & 4.69 \\
\hline Field operation & & 5.9 & 4.58 \\
\hline Digging trenches & & 6.0 & 4.61 \\
\hline Obstacle course & & 6.2 & 4.97 \\
\hline Assault course & & 6.9 & 5.22 \\
\hline Marching $\left(4 \mathrm{~m} \mathrm{~h}^{-1}\right)$ with a $27 \mathrm{~kg}$ load & & 8.2 & 6.87 \\
\hline Rapid marching & & 9.7 & 8.12 \\
\hline \multicolumn{4}{|l|}{ Energy expenditure of British soldiers in India } \\
\hline Standing at ease & 10 & 1.3 & 1.16 \\
\hline Standing at attention & & 1.4 & 1.25 \\
\hline Cleaning equipment & & 2.9 & 2.59 \\
\hline Signalling with morse, semaphore and lamp & & 3.0 & 2.68 \\
\hline Musketry training & & 3.2 & 2.86 \\
\hline Musketry-firing on range & & 3.8 & 3.39 \\
\hline Sentry duty & & 3.5 & 3.13 \\
\hline Squad drill-without arms & & 4.7 & 4.2 \\
\hline Squad drill with arms & & 4.8 & 4.29 \\
\hline Throwing grenades & & 4.7 & 4.2 \\
\hline Marching in drill order (load $13 \mathrm{~kg}$, speed $3.4 \mathrm{~m} \mathrm{~h}^{-1}$ ) & & 6.3 & 5.63 \\
\hline Bayonet exercises & & 6.7 & 5.98 \\
\hline Field exercises in extended order & & 7.8 & 7.0 \\
\hline Digging trenches & & 8.8 & 7.86 \\
\hline Horse clipping & & 4.2 & 3.61 \\
\hline Cleaning harness & & 4.8 & 4.13 \\
\hline Cleaning guns & & 5.1 & 4.39 \\
\hline Trotting on horseback & & 5.6 & 4.82 \\
\hline Cantering on horseback & & 6.4 & 5.51 \\
\hline Jumping on horseback & & 7.6 & 6.54 \\
\hline Harnessing and unharnessing & & 6.9 & 5.94 \\
\hline Grooming horses & & 8.3 & 7.14 \\
\hline \multicolumn{4}{|l|}{ Energy expenditure of US soldiers } \\
\hline Inspection & & 2.4 & 2.34 \\
\hline Fatigue duties & & 2.4 & 2.34 \\
\hline Drill & & 3.8 & 3.7 \\
\hline Digging foxholes (mixed with marching and short rest periods) & & 4.6 & 4.48 \\
\hline Mass games & & 5.2 & 5.06 \\
\hline Field march & & 5.5 & 5.35 \\
\hline Field march with rifle & & 6.5 & 6.33 \\
\hline Obstacle course with pack and rifle & & 6.6 & 6.43 \\
\hline Creeping and crawling with full equipment & & 7.9 & 7.69 \\
\hline Field march with rifle and $27-\mathrm{lb}$ pack at $3 \mathrm{~m} \mathrm{~h}^{-1}$ & & 8.0 & 7.79 \\
\hline Field march with heavy pack & & 8.9 & 8.66 \\
\hline \multicolumn{4}{|l|}{ Energy expenditure of Yugoslav soldiers } \\
\hline Dressing and undressing & & 2.5 & 2.11 \\
\hline Driving a tank & & 2.4 & 2.03 \\
\hline Adjusting caterpillar tracks & & 2.4 & 2.03 \\
\hline Cleaning a tank & & 2.8 & 2.37 \\
\hline Rifle exercises, lying down & & 2.8 & 2.37 \\
\hline Rifle exercises, kneeling & & 3.2 & 2.7 \\
\hline Taking off and putting on car tyres & & 3.3 & 2.78 \\
\hline Cleaning equipment & & 3.6 & 3.04 \\
\hline Cleaning gun & & 3.7 & 3.13 \\
\hline Rifle exercises, standing & & 3.8 & 3.21 \\
\hline Horse riding, slow & & 4.3 & 3.63 \\
\hline Cleaning horse & & 4.5 & 3.8 \\
\hline Lifting car by jack & & 4.5 & 3.8 \\
\hline Carrying boxes of ammunition & & 6.3 & 5.32 \\
\hline Horse riding, trotting & & 6.5 & 5.49 \\
\hline Digging a trench & & 8.0 & 6.76 \\
\hline Horse riding, galloping & & 8.1 & 6.84 \\
\hline
\end{tabular}

Abbreviation: PAR - physical activity ratio. 